CUAD. CONTAB. / BOGOTÁ, COLOMBIA, 15 (38): 459-500 / JULIO-DICIEMBRE 2014 / 459

\title{
Información financiera prospectiva: análisis comparado de su normalización y evidencia empírica*
}

doi: 10.11144/Javeriana.cc15-38.ifpa

\section{Eva María Ibáñez-Jiménez}

Profesora colaboradora, Departamento de Economía de la Empresa y Contabilidad, Facultad de Ciencias Económicas y Empresariales, Universidad Nacional de Educación a Distancia (UNED), España. Licenciada en Ciencias Económicas y Empresariales, (Sección Empresariales), Universidad Autónoma de Madrid (1997). Máster en Economía de los Servicios, Universidad Autónoma de Madrid, Departamento de Análisis Económico (2000). Doctora en Ciencias Económicas y Empresariales, Universidad Nacional de Educación a Distancia (2011).

Correo electrónico: eibanez@cee.uned.es

\begin{abstract}
Ángel Muñoz-Merchante
Catedrático de Economía Financiera y Contabilidad, Departamento de Economía de la Empresa y Contabilidad, Facultad de Ciencias Económicas y Empresariales, Universidad Nacional de Educación a Distancia (UNED), España. Licenciado en Ciencias Económicas y Empresariales (Sección Empresariales), Universidad Complutense de Madrid (1977). Doctor en Ciencias Empresariales, Universidad Nacional de Educación a Distancia (1989). Catedrático de Economía Financiera y Contabilidad (2001).

Correo electrónico: anmumer@cee.uned.es
\end{abstract}

\footnotetext{
* Artículo derivado de la tesis doctoral El Informe de gestión en el contexto internacional: un modelo de integración de la información corporativa, presentada en junio de 2011. Departamento de Economía de la Empresa y Contabilidad, Universidad Nacional de Educación a Distancia, España, UNED.
} 
Resumen Este artículo contribuye a mejorar el conocimiento de las prácticas divulgativas de información financiera prospectiva, mediante una revisión de la normativa contable internacional y de la evidencia empírica. En primer lugar, el análisis comparativo de la normalización de este tipo de información se aborda desde dos perspectivas. Por una parte, se identificaron los principios de adhesión voluntaria, recomendados por los más prestigiosos organismos contables del mundo en las guías internacionales para la elaboración del Informe de gestión de las compañías cotizadas (ASB, 2006; CA, 2009a; CNMV, 2013; FRC, 2013; IASB, 2010; SEC, 2003). Por otra, se revisaron las normas contables que exigen, por imperativo legal, información financiera prospectiva. Se eligieron tres casos de estudio: Estados Unidos, Reino Unido y Canadá, países que gozan de una mayor tradición en este ámbito. Posteriormente, se analizó la evidencia empírica que trata la información financiera prospectiva. Como resultado, se identificaron las metodologías aplicadas (análisis del contenido e índices de divulgación), las líneas de investigación abiertas en este campo y las principales conclusiones alcanzadas por los investigadores. Nuestro análisis confirma la utilidad de la información prospectiva para los inversores, así como sus limitaciones — escasez y sesgo en las previsiones-.

Palabras clave Información prospectiva; informe de gestión; información narrativa; análisis del contenido; índice de revelación

\section{Código JEL M41}

\section{Prospective Financial Information: Comparative Analysis of its Standardization and Empirical Evidence}

\footnotetext{
Abstract This article contributes to a better understanding of information dissemination practices of prospective financial information, by reviewing international accounting standards and empirical evidence. First, the comparative analysis of the standardization of this type of information is approached from two perspectives.
}

Next, the principles of voluntary membership, recommended by the most prestigious accounting bodies in the world in international guidelines for the preparation of the Management Report of listed companies were identified (ASB, 2006; CA, 2009a; CNMV, 2013; FRC, 2013; IASB, 2010; SEC, 2003). Furthermore, the accounting rules that legally required prospective financial information is reviewed. Three case studies were selected: US, UK and Canada, countries that enjoy a longer tradition in this field. Subsequently, the empirical evidence that the prospective financial information is analyzed. As a result, the methodologies applied (and indexes content analysis disclosure), open lines of research in this field and the main conclusions reached by researchers were identified. Our analysis confirms the usefulness of information to prospective investors and their - limitations and bias in their estimates-.

Keywords Prospective Information Management Report; narrative information; content analysis; disclosure index

\section{Informação financeira prospectiva: análise comparada da sua normalização e evidencia empírica}

Resumo Este artigo contribui para melhorar o conhecimento das práticas divulgadoras de informação financeira prospectiva, mediante revisão da normativa contábil internacional e da evidencia empírica. Em primeiro lugar, a análise comparativa da normalização deste tipo de informação é abordada desde duas perspectivas. De uma parte, identificaram-se os princípios de adesão voluntária recomendados pelos mais prestigiosos organismos contábeis do globo nas guias internacionais para a elaboração do Informe de gestão das companhias cotadas (ASB, 2006; CA, 2009a; CNMV, 2013; FRC, 2013; IASB, 2010; SEC, 2003). De outra, revisaram-se as normas contábeis que exigem informação financeira prospectiva, como um imperativo legal. Foram eleitos três casos de estudo: os Estados Unidos, Reino Unido e Canadá, países que gozam de maior tradição neste âm- 
bito. Posteriormente, analisou-se a evidência empírica que trata a informação financeira prospectiva. Como resultado, foram identificadas as metodologias aplicadas (análise de conteúdo e índices de divulgação), as linhas de pesquisa abertas neste campo e as principais conclusões conseguidas pelos pesquisadores. Nossa análise confirma a utilidade da informação prospectiva para os inversores, assim como suas limitações - escassez e viés nas previsões-.

Palavras-chave Informação prospectiva; informe de gestão; informação narrativa; análise de conteúdo; índice de revelação

\section{Introducción}

La información prospectiva es información sobre el futuro, sobre las perspectivas y planes de una entidad (IASB, 2013, pp. 141-142). Los reguladores de los mercados de valores definen la información prospectiva como aquella relativa a los posibles sucesos, condiciones, actuaciones o resultados de las operaciones, basada en hipótesis sobre las condiciones económicas futuras, incluyendo la información financiera prevista sobre los resultados, posición financiera o flujos de caja, presentada en forma de previsiones o estimaciones (CA, 2008a, p. 1; CNMV, 2013, p. 161). Por consiguiente, la información prospectiva es de muy diversa índole, tanto financiera en sentido estricto (elementos de los estados financieros expresados en términos previsionales obtenidos a partir de información histórica), como no financiera (procedente de la contabilidad interna; por ejemplo, estrategias y objetivos previstos, análisis de riesgos, presupuestos o indicadores de la gestión). La información prospectiva tiene carácter subjetivo, por lo que "su preparación requiere el ejercicio de juicio profesional” (IASB, 2010, Appendix). Además, puede presentarse en forma de estimaciones cuantitativas o de explicaciones cualitativas.

La información financiera prospectiva suele divulgarse en el Informe anual ${ }^{1}$, que forma parte de la denominada información narrativa (narrative reporting), término que carece de una definición precisa en la literatura contable. Se suele identificar por exclusión: la sección narrativa del Informe anual abarca todas sus páginas, salvo los estados financieros auditados (Deloitte, 2008, p. 1, p. 7). Su núcleo está constituido por el Informe de gestión ${ }^{2}$ (IG, en adelante), aunque también abarca otros documentos como la carta del presidente de la compañía, la introducción a los estados financieros, el resumen ejecutivo y otra información general sobre los empleados, la comunidad o los directivos (FRC, 2009, p. 56).

A diferencia de los estados contables - regulados detalladamente por normas como los Generally Accepted Accounting Principles, GAAP, los IFRS y/o las normas contables nacionales-, la regulación de la información prospectiva es escasa. La publicación de información prospectiva se exige en el IG, en virtud de las diferentes legislaciones nacionales mercantiles y de los mercados de valores; pero su contenido no se ha concretado, ni hay modelos oficiales en los que deba figurar. El nivel de exigencia varía

\footnotetext{
1 En sentido estricto, el Informe anual está compuesto exclusivamente por los estados financieros más el informe de gestión (IASB, 2005, p. 8).

2 Este informe recibe diferentes denominaciones: Management Commentary (IASB); Management Discussion and Analysis, MD\&A (IOSCO, Canadá y Estados Unidos); Management Report (Alemania), Strategic Report (Reino Unido); Review of Operations and Financial Conditions (Australia).
} 
de unos países a otros y de unas entidades a otras según su tamaño; se ha instaurado la práctica generalizada de establecer legalmente el contenido mínimo en el IG y desarrollarlo mediante guías de adhesión voluntaria, basadas en principios y mejores prácticas. En consecuencia, se suele considerar que la información prospectiva es voluntaria o pseudovoluntaria.

De ahí el interés del International Accounting Standards Board, IASB, en la estandarización del IG y de la información prospectiva que contiene, en línea con las propuestas de otros organismos internacionales (SEC, CA, ASB, GASB, G100) y los recientes avances en su regulación (Companies Act, Regulations 2013, Reino Unido). Ese interés justifica la primera parte de este trabajo. En ella, se presenta un análisis comparado de esta información - compleja por su propia naturaleza, subjetiva y voluntaria-dividido en sus dos vertientes: obligatoria en la legislación mercantil y del mercado de valores, por una parte, y voluntaria, en las guías de buenas prácticas sobre el IG, por otra. En esta primera parte se emplea un método descriptivo de las normas internacionales, en el marco de un análisis comparativo, que desarrolla tres casos de estudio en particular: Estados Unidos, Reino Unido y Canadá.

El grueso de la investigación contable se centra en el análisis de los datos de los estados financieros, mientras que la investigación sobre la denominada información narrativa o voluntaria, que acompaña esos estados, está en una fase de desarrollo incipiente. La sistematización de las investigaciones en el ámbito de la información narrativa es escasa (Ahmed \& Courtis, 1999; Jones \& Shoemaker, 1994; Marston \&
Shrives, 1991). Dado que no hemos encontrado ningún trabajo previo de esas características, centrado en la información narrativa de carácter prospectivo, en la segunda parte de este artículo se ha realizado una revisión de la evidencia empírica centrada en la información prospectiva contenida en los informes anuales de las sociedades cotizadas de diferentes países.

Nuestros criterios de selección de los trabajos tienen una doble perspectiva. En primer lugar, las investigaciones analizadas siguen alguna de las metodologías definidas por Vivien Beattie, Bill McInnes y Stella Fearnley (2004) como las propias de la información financiera de carácter narrativo. En segundo lugar, la selección está restringida a los análisis sobre información prospectiva extraída directamente del informe financiero anual y se excluyen aquellos otros que utilizan información no contable o no financiera (como la información social o medioambiental) o información no obtenida directamente del Informe anual (como bases de datos de previsiones de analistas financieros o de gestores).

El contenido del presente trabajo se divide en dos partes. La primera parte - cuyo objetivo es comparar, desde un punto de vista normativo, los requisitos que se exigen en la publicación de la información prospectiva- está compuesta por dos apartados. En un primer apartado de carácter introductorio, se sitúa la información prospectiva en el contexto de la información contable y se exponen sus beneficios y limitaciones (segundo apartado). El siguiente apartadotercero - se ha dividido en dos subapartados. El primero consiste en un análisis de los estándares contables o guías sobre el IG, de cumplimiento 
voluntario en su mayoría, que señalan los principios que deben sustentar la elaboración de información prospectiva. Posteriormente - apartado 3.2- se compara la regulación sobre información prospectiva en Estados Unidos, Reino Unido y Canadá, países en los que esa información ha alcanzado un mayor grado de desarrollo.

En la segunda parte del artículo — cuarto apartado—se sistematiza la evidencia empírica sobre información prospectiva, a fin de identificar las metodologías utilizadas, los temas objeto de estudio y las principales conclusiones alcanzadas. El objetivo último de esta revisión es determinar si su divulgación puede entenderse como un instrumento de transparencia hacia el mercado, esencial para dar a conocer información relevante no recogida en los estados financieros o, por el contrario, como un instrumento publicitario que distorsiona la objetividad de las cifras contables.

\section{Divulgación de la información financiera prospectiva en el informe de gestión}

\subsection{Relación entre información financiera prospectiva e informe de gestión}

Los estados financieros se han demostrado insuficientes para cubrir las necesidades informativas del mercado. El IASB (Marco conceptual, párr. 13) ha reconocido que esos estados no bastan para conducir las decisiones económicas de los usuarios, por cuanto estos persiguen el establecimiento de perspectivas racionalmente fundadas sobre los flujos de caja que esperan recibir. En consecuencia, prestigiosos organismos contables han elaborado modelos orientados a mejorar la calidad de toda la información corporativa que las sociedades lanzan al mercado, al margen de los estados financieros, en los que propugnan que esa información debe ofrecer un enfoque prospectivo (AICPA, 1994; EBRC, 2005; FASB, 2001; ICAEW, 2003a; IIRC, 2013).

Ya en los años ochenta, la Securities and Exchange Commission, SEC (1980, Release 33-6234) manifestaba la necesidad de una explicación narrativa adicional a los estados financieros, que permitiera a los inversores juzgar la calidad de los beneficios y la probabilidad de que la gestión histórica fuera indicativa de la gestión futura. Los reguladores de todo el mundo exigen que, junto a los estados contables, se presente el IG, que consiste en un análisis -basado en indicadores - de la evolución del negocio o gestión desempeñada por la compañía y sus perspectivas. Ese análisis contiene lo que se denomina información no financiera, otra información financiera o información voluntaria, e incluye información prospectiva. El IASB y otros organismos internacionales reconocen el carácter complementario del IG respecto a los estados financieros, por su capacidad interpretativa y predictiva. Así mismo, consideran que el IG presentado en el informe financiero anual (financial report) forma parte sustancial de la información financiera o contable (IASB, 2005, p. 8).

El IG se ha ido configurando durante el pasado decenio (2003-2013) como el principal complemento de la información comunicada en los estados financieros, al comprender información estratégica procedente de la dirección: 
análisis de las operaciones, información prospectiva y sobre riesgos (Muslu, Radhakrishnan, Subramanyam \& Lim, 2011, p. 1, p. 5). La SEC y sus homólogos defienden hoy la importancia del IG por la orientación prospectiva de su análisis, reafirmando su valor adicional a los estados financieros. Este informe se ha convertido en un elemento central en la comunicación financiera externa (CA, 2009a, p. 7).

Compañías de todo el mundo están adoptando progresivamente un enfoque prospectivo en sus informes financieros, bien en cumplimiento de los requisitos legales mercantiles y de los mercados de valores internacionales sobre el IG, o bien voluntariamente. Para los inversores, la información prospectiva es uno de los contenidos más valiosos del Informe anual, aunque no esté auditada al mismo nivel que los estados financieros, porque facilita la toma de sus decisiones, basadas no solo en información histórica, sino en las previsiones de los propios gestores sobre los objetivos de la entidad (ABI 2006, p. 3; $\mathrm{ACCA}^{3}, 2010$, p. 8, p. 20; CIMA, PwC, Radley Yeldar \& Tomkins, 2006, p. 14; Copeland, 1978, p. 4; FASB, 2010, QC2; Independent Audit Limited, 2006, p. 11; ICAEW, 2003b, p. 8; IASB, 2010, BC2 y BC3; OCDE, 2004, p. 50).

Aparte de la información prospectiva que se encuentra en el IG, las empresas pueden pu-

3 El 64\% de los 231 directivos de grandes compañías internacionales entrevistados en este estudio, concibe la información prospectiva como uno de los contenidos críticos para los accionistas, junto a los siguientes, de entre los que figuran en las secciones narrativas del Informe anual: los resultados y posición financiera (87\%), los riesgos y su gestión (67\%), la descripción del modelo de negocio (60\%) y los indicadores clave (58\%). blicar alguna información de este tipo en otros lugares, por ejemplo, las sociedades cotizadas suelen incluir información prospectiva en los anuncios de sus resultados.

\subsection{Beneficios y limitaciones de la información prospectiva}

El Financial Accounting Standards Board, FASB, y otros organismos han identificado los beneficios y costes genéricos de la información voluntaria, cuya definición ${ }^{4}$ incluye la información prospectiva (CA, 2009a, p. 17; CICA, 2002, p. 13, p. 19; G100, 2003, p. 7). Entre los primeros están la reducción de los costes de capital y de la deuda, mayor demanda y liquidez de los títulos en el mercado, e interés para inversores institucionales y analistas financieros (Botosan, 1997; Botosan \& Plumlee, 2002; Healy, Hutton \& Palepu, 1999; Lang \& Lundholm, 1996; Sengupta, 1998; Welker, 1995).

No obstante, los administradores suelen mostrarse reacios a publicar información prospectiva. Muchos evitan realizar predicciones y prefieren que los usuarios hagan las suyas propias, a partir de la información histórica, a causa de los costes asociados a su carácter pseudovoluntario: pérdida de ventajas competitivas por revelar datos sensibles, costes legales derivados de la responsabilidad de los administradores sobre la información que emiten y costes inherentes a su elaboración (ACCA 2006, p. 4;

4 El FASB define información voluntaria como aquella que los estados financieros no contienen y que no es requerida por los GAAP o por las normas de la SEC. No obstante, el FASB reconoce que gran parte de lo que define como información voluntaria se solicita obligatoriamente por la SEC cuando esta requiere el MD\&A en los formatos oficialmente establecidos (FASB, 2001, p. 5). 
Aljifri \& Hussainey, 2007, p. 883; Gelb, Holtzman \& Mest, 2008, p. 244). Dado que este tipo de información está sujeta a incertidumbre, parece lógico que los administradores se limiten a cumplir la obligación formal de presentarla, sin adecuarse a su verdadero objetivo, lo cual les permite eludir los datos de carácter estratégico más comprometidos (Mallo, 1990, pp. 38-39).

Por otra parte, la experiencia de muchos países muestra que el IG puede exponer asuntos significativos para el futuro de la entidad, al explicar las tendencias e incertidumbres que afronta, sin comprometer la confidencialidad del negocio (G100 2003, p. 8, p. 11) (a favor, ABI 2006, p. 4; CNMV 2013, p. 47; ICAC 2002, p. 151; PwC, 2007, pp. 6-8). Por ello, se han arbitrado algunas soluciones de los problemas inherentes a la información prospectiva:

1. Establecer legalmente cláusulas de no responsabilidad o cautela (safe harbour provisions) sobre la información prospectiva, que debe ser previamente separada de la información auditable. Las cláusulas de protección eliminan la responsabilidad legal de quienes las emplean, bajo la presunción de buena fe (IASB, 2009, BC10d). Este sistema de cláusulas fue creado por la SEC en 1979 para proteger las estimaciones del MD\&A $\mathrm{A}^{5}$ estadounidense y otorgarle mayor importancia a la relevancia de la información que a la pérdida potencial de fiabilidad (Schroeder, Clark \& Cathey, 2005, p. 551).

5 Las cláusulas safe-harbour protegen a los emisores en relación con la información prospectiva del MD\&A, si tal información tiene una base razonable y se elabora de buena fe (Rule 175, Securities Act; Rule 3b-6; Securities Act Release 6084, June 25, 1979; Regulation S-K, Item 303, instructions to paragraph a. 7.).
A pesar de la existencia de estas cláusulas, la propia regulación del MD\&A y los riesgos legales limitan el potencial comunicativo de este informe, según entrevistas realizadas a directivos financieros de sociedades cotizadas en Estados Unidos, Canadá y Reino Unido (Tarca, Street \& Aerts, 2011, p. 53).

2. En muchos países, es frecuente que el IG se someta a revisión por parte de un asesor jurídico - interno o externo-que determine si cumple la legislación del mercado de valores vigente en su país en relación con la información prospectiva (Chlala \& Girard 1993, pp. 34-35).

Los estudios profesionales realizados por grandes consultoras y organismos contables, en su mayoría británicos, referidos a la información narrativa contenida en el Informe anual, esencialmente en el IG, ponen de manifiesto las consecuencias de las limitaciones expuestas, sobre la praxis informativa.

Esos estudios señalan que, a pesar de que ha aumentado sustancialmente durante los últimos años, la presencia de información prospectiva en los informes anuales es uno de los contenidos susceptibles de mejorar (junto con la información sobre riesgos y los recursos no financieros). Su cantidad y calidad varían; la comunicación de información prospectiva cuantitativa es poco común, en especial entre las empresas cotizadas de menor tamaño. El análisis de los factores con incidencia en la gestión futura está presente en casi todos los informes de las grandes cotizadas británicas o canadienses - entre 86 y 95\%-pero menos de un tercio exhibe información de calidad, 
basada en tendencias precisas y/o en objetivos cuantitativos. Un porcentaje reducido - entre 10 y $20 \%$-, pero creciente, presenta indicadores prospectivos e información sobre inversiones previstas (ABI, 2006; ASB, 2008, 2009b; Black Sun, 2009; DBIS, 2010; Deloitte, 2008, 2009, 2010, 2011; OSC, 2008, 2013; PwC, 2008; Radley Yeldar, 2008a, 2008b; Tomorrow's Company, 2007).

La incertidumbre, acentuada por la crisis financiera internacional, se considera un impedimento para que los gestores emitan información previsional (ASB, 2009a, p. 3; CA, 2009b, p. 22, p. 26; Deloitte, 2009, p. 50; PwC, 2009 , p. 3). Pero en un contexto de crisis, las previsiones sobre los resultados y la liquidez, junto con un análisis de sensibilidad, cobran, si cabe, mayor relevancia, en especial si cuestionan el principio de empresa en funcionamiento (ACCA, 2010, pp. 8-9, pp. 23-25; CA, 2008c, 2009b; FRRP, 2010, p. 1).

Por último, la auditoría externa del IG se percibe como un inconveniente de la información prospectiva, por las dificultades que presenta. Prestigiosos organismos contables han trabajado durante años sobre la adaptación al IG de las características cualitativas del marco conceptual de la información financiera (ASB, 2006, pp. 4-25; CA, 2009a, pp. 25-28; CICA, 2002, pp. 26-30; G100, 2003, p. 10-11; GASB, 2005, pp. 9-35; IASB, 2005, pp. 58-95). Publicado el nuevo marco conceptual del FASB (2010), el IASB, en lugar de adaptar las características cualitativas al IG, ha establecido que este informe queda dentro del alcance de ese marco, y que comparte el objetivo, los usuarios y las caracterís- ticas cualitativas de la información financiera (IASB, 2010, p. 20, BC42). Por tanto, incluso con limitaciones, estas le son aplicables, incluso la verificabilidad, dado que entre otras, la información prospectiva del IG puede ser auditada por expertos independientes (IASB, 2010, BC44).

Internacionalmente, la auditoría del IG se limita a la verificación de la concordancia de los datos de este informe con los recogidos en los estados financieros, pero no alcanza al resto del contenido del informe, y, por consiguiente, a la información prospectiva que ofrezca. Así, en Estados Unidos y Canadá, a este tipo de información se le aplica el estándar de auditoría International Standard on Auditing (ISA) 720 de la International Federation of Accountants (IFAC), sobre la responsabilidad del auditor en relación con otra información incluida en documentos que acompañan las cuentas anuales auditadas.

En 2012, este organismo publicó el estándar ISAE 3400, para los encargos sobre el examen de la información financiera prospectiva con carácter cuantitativo (no narrativo). En 2001, el AICPA había emitido el estándar de auditoría AT Section 701: Management's Discussion and Analysis, basado en procedimientos específicos adaptados a los contenidos del MD\&A (AICPA, 2001). A pesar de la existencia de estos estándares, es escasa la demanda de trabajos de opinión separada sobre el MD\&A o la información prospectiva (IASB, 2005, p. 59), por su propia naturaleza previsional y subjetiva. 


\section{Normalización de la información prospectiva}

\subsection{Análisis de los estándares de carácter voluntario}

\subsubsection{Las guías sobre el IG y la información prospectiva}

Objeto de continua revisión, el IG forma parte de la agenda de los organismos internacionales emisores de normas contables al más alto nivel, que han reconocido su importancia y consecuentemente, han publicado guías para su elaboración de seguimiento voluntario por lo general $^{6}$ (ASB, 2006; CA, 2009a; CNMV, 2013; FRC, 2013; G100, 2003; GASB, 2005; IASB, 2010; IOSCO, 2003; SEC, 2003). Su finalidad es proporcionar a los gestores de las sociedades cotizadas un conjunto de principios que clarifiquen el contenido del IG, regulado en el ámbito internacional con distinto grado de detalle en las diferentes jurisdicciones. $\mathrm{Al}$ mismo tiempo, la normativa del IG ha evolucionado tanto en la Unión Europea (Directivas 2003/51/ CE; 2004/25/CE; 2004/109/CE; 2006/46/CE; 2013/34/UE) - en especial en el Reino Unido, (Regulaciones de 2005; Companies Act 2006; Regulaciones de 2013) -, como en Estados Unidos y Canadá, pioneros en este ámbito, donde se vienen realizando desarrollos específicos sobre determinados contenidos del MD\&A, entre ellos, la información prospectiva (apartado 3.2).

6 Los requisitos del IG en Alemania son los que imponen las directivas europeas, pero adicionalmente las empresas matrices de los grupos de sociedades deben cumplir el estándar contable sobre el IG, elaborado por el German Accounting Standards Board (GASB) GAS No. 15: Management Reporting.
Según las guías internacionales de buenas prácticas, los gestores deben explicar en el IG la situación y el rendimiento pasado y su evolución previsible, mediante un análisis financiero y no financiero basado en indicadores, que muestre el progreso alcanzado en los objetivos de la entidad, de modo que el informe cumpla las siguientes funciones (FRC, 2013, p. 16; IASB, 2009, p. 11):

- Interpretar los estados financieros en el contexto o entorno en el que opera la entidad.

- Conocer la visión de los gestores sobre los principales asuntos que afronta y cómo van a ser gestionados.

- Valorar las estrategias adoptadas y la probabilidad de que tengan éxito.

Entre los organismos emisores de las guías hay un alto grado de consenso en relación con el contenido del informe, que debe contemplar los siguientes aspectos (ASB 2006, p. 27; CA, 2009a, p. 31; CNMV, 2013, pp. 20-25; IASB, 2005, p. 100; 2010, p. 24, BC48; SEC, 2003, IIIB):

- Naturaleza del negocio.

- Objetivos y estrategias de los gestores para lograr sus objetivos.

- Principales recursos, riesgos y relaciones de la entidad.

- Resultados actuales y previstos de las operaciones.

- Medidas críticas de la gestión utilizadas por los gestores para evaluar el logro de los objetivos.

En resumen, el IG es un documento que informa sobre dónde compite la empresa (entorno), cuáles son sus objetivos (estrategias), con 
qué recursos cuenta para conseguirlos (factores clave financieros y no financieros), qué riesgos reales soporta, cómo se ha gestionado (indicadores clave) y qué espera (información prospectiva). Así mismo, explica por qué la posición lograda por una empresa difiere de la posición esperada en relación con las estrategias que se adoptaron para lograr sus objetivos, así como sus futuras estrategias y perspectivas.

\subsubsection{Principios generales que} sustentan la elaboración de información prospectiva, según las guías internacionales de buenas prácticas

Todas las guías establecen entre otros principios fundamentales que el IG debe estar orientado al futuro ${ }^{7}$. Por consiguiente, todos sus contenidos deben tener carácter prospectivo. El equilibrio entre el análisis histórico y futuro es clave en el IG (DTI 2004, p. 11).

Así, los organismos supervisores de las bolsas internacionales y otros organismos contables normalizadores proponen que el IG constituya una explicación elaborada por los gestores, en la que identifiquen, a partir de toda la información histórica financiera y no financiera, los factores que a su juicio hayan incidido en la posición financiera y los resultados de la empresa durante

7 Aunque con matices, el análisis comparado de las diferentes guías internacionales sobre el IG muestra un alto grado de consenso sobre los principios y características cualitativas que debe poseer. Entre los primeros se encuentran (IASB 2010, p. 12-13):

- Carácter complementario respecto a los estados financieros.

- Análisis de los estados financieros desde el punto de vista de la dirección (carácter estratégico).

- Ofrece información sobre el futuro de la empresa (carácter prospectivo). el período cubierto por los estados financieros, así como las tendencias e incertidumbres que explican la evolución, calidad y variabilidad previsible de los resultados y de los flujos de caja (ASB, 2006, p. 8; CA, 2009a, p. 26; CNMV, 2013, p. 18; FRC, 2013, p. 18 y p. 22; GASB, 2005, p. 3; G100, 2003, p. 9; IASB, 2010, p. 18, BC27; IOSCO, 2003, p. 2; SEC, 2003, III.B.4). De este modo, los inversores pueden anticipar la probabilidad de que la gestión realizada sea indicativa de la gestión futura.

En resumen, proporcionar información prospectiva significa (ASB 2006, p. 8; CA, 2009a, p. 40, pp. 42-46; CNMV, 2013, p. 18, p. 46; IASB, 2010, p. 18; PwC, 2007, p. 5):

- identificar las tendencias, factores, oportunidades y riesgos relevantes para la valoración de la gestión realizada (rendimiento pasado) y futura (evolución previsible), por su impacto sobre la posición financiera y los resultados previstos.

- comunicar el progreso alcanzado en los objetivos del negocio a largo plazo y valorar anticipadamente los resultados esperados de las estrategias adoptadas.

La manera generalizada de presentar el análisis consiste en explicar, uno por uno, la evolución pasada y prevista de cada contenido: objetivos, recursos, riesgos, relaciones, liquidez, resultados, etc. No obstante, la guía alemana - obligatoria para las sociedades matrices de los grupos consolidados-requiere que la información prospectiva se publique en un apartado específico. Además, concreta el período de previsión informativo en dos años (GASB 2005, p. 34, p. 87 , p. 91). 


\section{INFORMACIÓN FINANCIERA PROSPECTIVA / E. IBÁÑEZ, Á. MUÑOZ / 469}

En especial, las guías inciden en la discusión en términos previsionales de las estrategias y objetivos previstos para la entidad en su conjunto y sus principales negocios, y el análisis de los resultados (CNMV, 2013, p. 31; IASB, 2010, p. 17). Los objetivos deben explicarse en el contexto estratégico a largo plazo y, en el caso de que se cuantifiquen, indicarse su variabilidad, para lo que se pueden utilizar intervalos o rangos que comuniquen el riesgo o elasticidad de los números (CA, 2009a, pp. 43-44). Es frecuente que las guías requieran información sobre las políticas o decisiones estratégicas y tácticas, como la introducción de nuevos productos y procesos o su expansión hacia nuevos mercados, así como el análisis de las inversiones previstas, en especial los esfuerzos en $\mathrm{I}+\mathrm{D}$, incluyendo su detalle por segmentos, proyectos, su contribución en las actividades del negocio y su financiación (ASB, 2006, p. 48; CNMV, 2013, p. 39, p. 48; GASB, 2005, p. 84, pp. 121123; G100, 2003, p. 17).

En el IG, los gestores han de complementar el análisis de los resultados históricos con el de los resultados previstos y han de señalar hasta qué punto los primeros son indicativos de los segundos. Deben expresar sus expectativas, tanto positivas como negativas, sobre los resultados y la posición financiera de la entidad y sus segmentos. Se recomienda el análisis de las tendencias en los gastos e ingresos, y explicar las diferencias entre el resultado de la explotación con el beneficio neto (CA, 2009a, p. 39; CNMV, 2013, p. 32; GASB 2005, pp. 89-90).

Puesto que por su naturaleza no puede ser verificada objetivamente, la información prospectiva presentada por los gestores "de buena fe" debe identificarse expresamente, al distinguirla del resto con claridad y emplear un lenguaje específico que alerte a los usuarios sobre la incertidumbre propia de las previsiones (CA, 2009a, p. 44; GASB, 2005, p. 15, p. 86).

Dado su carácter confidencial, las guías no exigen cuantificar los resultados, los objetivos o los indicadores previstos. Se trata de explicar las tendencias, factores e incertidumbres relevantes para el futuro de la compañía, sin anticipar necesariamente las previsiones de los resultados que provocarán tales incertidumbres (CNMV, 2013, p. 24; DRSC, 2006, p. 6; FRC, 2013, p. 22; G100, 2003, p. 6; GASB, 2005, p. 34; IASB, 2010, p. 10, BC38, BC40). Las tendencias del mercado, las oportunidades y riesgos del negocio o las prioridades estratégicas a largo plazo también proporcionan a los inversores las señales que necesitan para tomar sus decisiones (Independent Audit Limited, 2006, p. 9; PwC, 2007, pp. 6-7; Radley Yeldar, 2008b, p. 13).

Es recomendable la cuantificación de los objetivos financieros y no financieros (GASB, 2005, p. 120). En tal caso, para garantizar su fiabilidad, los gestores deben explicar la metodología empleada para calcularlos (modelos de previsión). Deben valorar también el impacto potencial de cambios significativos en las hipótesis asumidas, al identificar los factores, riesgos o incertidumbres que inciden en la variabilidad de los resultados y explicar los intervalos de estimaciones posibles (ASB, 2006, p. 10; CA, 2009a, p. 45; CNMV, 2013, p. 27, p. 47; GASB, 2005, p. 17, p. 86; IASB, 2005, p. 57, p. 76; 2009, p. 35; 2010, p. 18 у BC39).

Si finalmente se materializa un suceso probable o si las hipótesis en que se basa la 
información prospectiva son desechadas, las previsiones realizadas y los resultados obtenidos diferirán. Esta circunstancia ha de comunicarse, bien en el IG intermedio, bien en el anual, con la justificación de las desviaciones producidas (ASB, 2006, p. 12; CA, 2009a, p. 46; GASB, 2005, p. 16, p. 33; IASB, 2009, pp. 17-19; 2010, p. 18-19, IN3).

\subsection{Análisis de la regulación comparada: últimos avances}

Al margen de los principios o recomendaciones estudiados en el apartado anterior, los reguladores internacionales exigen, por imperativo legal, información prospectiva por medio del IG. En los extractos que figuran en la tabla 1, se resumen esos requisitos mínimos y se muestran las cláusulas de cautela (safe harbour provisions) sobre la información prospectiva emitida en el IG. Los reguladores de cada país han de estudiar la necesidad de establecer este tipo de cláusulas en su caso particular, para dotar los informes de mayor flexibilidad e innovación (IASB, 2009, BC10).

En este apartado, estudiaremos la regulación de la información prospectiva en tres casos: Estados Unidos, principal referente en esta cuestión; Reino Unido y Canadá, por haber acometido recientes modificaciones.

\section{Legislación internacional sobre la información prospectiva en el IC}

\begin{tabular}{|c|c|c|}
\hline $\begin{array}{l}\text { Unión } \\
\text { Europea }\end{array}$ & $\begin{array}{l}\text { Directiva 2013/34/UE, art. } 19 \\
\text { Informe de Gestión }\end{array}$ & $\begin{array}{l}\text { 1. El Informe de gestión deberá contener al menos una imagen fiel de la } \\
\text { evolución de los negocios, los resultados y la situación de la empresa, junto } \\
\text { con una descripción de los principales riesgos e incertidumbres a los que se } \\
\text { enfrenta... } \\
\text { 2. El informe incluirá así mismo: a) la evolución previsible de la sociedad (...) }\end{array}$ \\
\hline España & $\begin{array}{l}\text { Ley de Sociedades de Capital, } \\
\text { art. } 262 \\
\text { Código de Comercio, art. } 49 \\
\end{array}$ & $\begin{array}{l}\text { El Informe de gestión habrá de contener una exposición fiel sobre la evolución } \\
\text { de los negocios y la situación de la sociedad, junto con una descripción de los } \\
\text { principales riesgos e incertidumbres a los que se enfrenta. }\end{array}$ \\
\hline Reino & Companies Act 2006, & (2) El Informe estratégico (Strategic Report) debe contener: \\
\hline Unido & $\begin{array}{l}\text { Regulations } 2013 \\
414 \mathrm{C} \\
\text { Strategic Report }\end{array}$ & $\begin{array}{l}\text { una imagen fiel del negocio, y } \\
\text { una descripción de los principales riesgos e incertidumbres que afronta la sociedad. } \\
\text { (4) En el caso de una sociedad cotizada, el Informe estratégico debe incluir: } \\
\text { (a) una descripción de la estrategia de la sociedad, (b) una descripción del } \\
\text { modelo de negocio de la sociedad, (c) los principales factores y tendencias que } \\
\text { probablemente afectarán el futuro desarrollo, gestión y posición financiera del } \\
\text { negocio de la compañía y (...) }\end{array}$ \\
\hline & $\begin{array}{l}\text { Cláusulas de protección } \\
\text { Confidencialidad 414C }\end{array}$ & $\begin{array}{l}\text { (12) En el Informe estratégico no es obligatorio informar sobre desarrollos } \\
\text { inminentes o asuntos en curso de negociación, si la información, en opinión } \\
\text { de los administradores, puede perjudicar seriamente los intereses de la } \\
\text { compañía. }\end{array}$ \\
\hline & Safe harbour, 463 & $\begin{array}{l}\text { (3) El administrador solo será responsable si sabía que el informe era falso o } \\
\text { erróneo, si fue imprudente al respecto o si conocía que omitía información } \\
\text { deliberadamente. }\end{array}$ \\
\hline
\end{tabular}




\section{Legislación internacional sobre la información prospectiva en el IG}

\begin{tabular}{ll}
\hline Estados & Regulation S-X, Item 303 \\
Unidos & MD\&A of Financial Condition \\
SEC & and Results of Operations
\end{tabular}

Las compañías deben identificar "las tendencias conocidas, demandas, acuerdos, sucesos o incertidumbres que provocarán o que razonablemente provocarán un aumento o disminución material de la liquidez, (...) los recursos de capital (...) las ventas o ingresos de las operaciones continuas".

El análisis se centrará en los sucesos e incertidumbres materiales (...) (A) que podrían tener un impacto en las operaciones del futuro, aunque no lo hayan tenido en el pasado y (B) que hayan tenido un impacto en las operaciones de las que ya se haya informado (pasadas), aunque no se espere que tengan un impacto en el futuro (Instrucciones al párrafo a) 3.).

Cláusula safe harbour Cualquier tipo de información prospectiva que se ofrezca estará expresamente cubierta por la cláusula safe harbour (Rule 175 Securities Act, Rule 3b-6 Exchange Act and Securities Act Release 6084, June, 1979).

\begin{tabular}{ll}
\hline Canadá & National Instrument 51- \\
CSA & 102F1: MD\&A
\end{tabular}

Part 1 (g) Información prospectiva: el MD\&A requiere una discusión de las tendencias, sucesos, acuerdos u operaciones e incertidumbres conocidas que afecten con probabilidades razonables el negocio (liquidez, recursos financieros y resultados). Sin embargo, el MD\&A no requiere que la compañía realice previsiones detalladas sobre los ingresos, gastos u otra información.

Cláusula safe harbour Hay una cláusula safe harbour para la información prospectiva en el régimen de responsabilidad civil que se aplica a los informes de los mercados secundarios. Este régimen requiere varias condiciones:

- Primera, adoptar un lenguaje preventivo y advertir que los resultados previstos pueden diferir de los reales.

- Segunda, que se expongan los factores materiales o hipótesis relacionados con la información prospectiva.

- Que dichas hipótesis o factores materiales sobre los que descansa la información prospectiva estén "próximos" a esta información.

En ausencia de una definición legal de "próximo", los abogados aconsejan a sus clientes establecer una referencia cruzada desde la información prospectiva hacia el estado que advierte de la existencia de esa información y de las hipótesis en que se basa, que suele figurar al comienzo del MD\&A (CA, 2008a, p. 6).

Tabla 1. Legislación internacional sobre la información prospectiva en el IG Fuente: elaboración propia

\subsubsection{Estados Unidos}

La SEC ha hecho hincapié en la necesidad de incluir información prospectiva en el MD\&A, al objeto de proporcionar en una sección "la información histórica y prospectiva que permite a los inversores y otros usuarios valorar la situación financiera y resultados de las operaciones de la empresa emisora, con particular interés en las perspectivas de la entidad para el futuro" (SEC 1989, III.A).
La legislación de la SEC sobre el MD\&A exige a las sociedades cotizadas explicar los sucesos e incertidumbres materiales que puedan hacer que la información de los estados financieros presentados no sea necesariamente indicativa de la situación financiera y de los resultados futuros (SEC, 1982, Item 303, a1, a2, a3). Por ello, al analizar la liquidez, los recursos financieros y los resultados deben identificar "las tendencias conocidas, demandas, acuerdos, 
sucesos o incertidumbres que provocarán o que razonablemente provocarán un aumento o disminución material de los mismos" (tabla 1).

En 1989, la SEC emitió un pronunciamiento, Management's Discussion and Analysis of Financial Condition and Results of Operations; Certain Investment Company Disclosure, que trataba específicamente la información prospectiva. Según esta interpretación de la normativa general, se distinguen dos tipos de información prospectiva: obligatoria y voluntaria. La primera es aquella que está basada o se puede derivar de las tendencias, sucesos e incertidumbres conocidas en el presente (tendencias conocidas), que se espera razonablemente que tengan efectos materiales (por ejemplo, una reducción en los precios de producción de la empresa informante o en las unidades vendidas de un determinado producto, o una disminución en su cuota de mercado). La segunda es aquella basada en la anticipación o predicción de una tendencia, suceso o incertidumbre (tendencias anticipadas). Las cláusulas safe harbour se aplican a ambos tipos de información.

Las empresas estadounidenses tienen obligación de informar cuando la tendencia o incertidumbre es conocida en el presente por los gestores y con probabilidades razonables tendrá efectos en la situación financiera o resultados futuros. Ejemplos de tendencias conocidas que afectan las operaciones futuras son: la caída de los precios de los productos de la empresa informante, la caída de la cotización de sus acciones, la posibilidad de que no se renueve un contrato con proveedores o la entrada en vigor de una legislación nueva. Por ejemplo, si se conoce que se ha producido un incremento de la demanda, debe explicarse el presupuesto de inversiones y su financiación correspondiente, necesarios para mantener el crecimiento de las ventas, incluso aunque no haya acuerdos financieros legalmente establecidos. También se debe informar sobre las inversiones o los gastos presupuestados necesarios para financiar un nuevo producto o línea de negocio cuyo lanzamiento se haya anunciado públicamente (SEC, 1989, III.B).

En consecuencia, los gestores deben, primero, revisar cuidadosamente los datos conocidos sobre los factores que afecten la liquidez, las inversiones y los resultados. Y, segundo, valorar (SEC1989, III.B):

1. Si la tendencia, demanda, suceso, contrato o incertidumbre va a suceder con probabilidades razonables. En caso negativo, no es necesario informar.

2. Si no pueden llegar a esa conclusión, deben evaluar objetivamente las consecuencias de la tendencia, demanda, etc., asumiendo que sucederá, e informar de ello.

Aunque estas exigencias tienden a ser específicas, el formato de presentación es flexible. Debemos destacar que la regulación estadounidense, al igual que la de otros países como Canadá o Reino Unido, no obliga a establecer previsiones, aun cuando exige que se expongan los sucesos conocidos en el presente que puedan tener efectos materiales en el futuro. En resumen, se solicita que se explique el impacto potencial de sucesos o factores bajo diferentes escenarios razonables, pero no es necesario que se cuantifiquen los resultados. Por tanto, se puede afirmar que, hasta cierto punto, la infor- 
mación prospectiva es voluntaria. No obstante, la SEC ha sancionado ${ }^{8}$ a las compañías cuando han ocultado tendencias conocidas sobre los resultados o la liquidez.

\subsection{Reino Unido}

En el Reino Unido, todas las sociedades, cotizadas y no cotizadas - salvo las calificadas como pequeñas-deben elaborar el Strategic Report - documento equivalente al IG español-, cuyo contenido se ha modificado recientemente: nueva sección 414, de la Companies Act 2006, que ha reemplazado la sección 417, Business Review (BR), denominación anterior del informe. En la tabla 2 se han sintetizado los contenidos mínimos que exige el legislador británico en el Strategic Report desde 2013. Se destacan en cursiva los que conciernen a la información prospectiva.

Inicialmente, la legislación mercantil británica requería en el Directors' Report (Companies Act 1985, Section 234) una indicación de la evolución esperada de la compañía, pero históricamente las empresas cubrían este requisito con un breve comentario sobre el nivel probable de actividad previsto para el año siguiente (Deloitte, 2006, p. 2). Posteriormente, el contenido del IG en Reino Unido (Business Review, BR) se vio incrementado de acuerdo con las exigencias comunitarias, en particular con lo dispuesto por la Directiva 2003/51/CE que requería "una exposición fiel sobre la evolución

8 Pueden consultarse a modo de ejemplo, los expedientes sancionadores de la SEC contra Caterpillar (SEC, 1992), Sony (SEC, 1998) o Coca-Cola (SEC, 2005) por no informar adecuadamente en el MD\&A de la sociedad matriz, acerca del impacto en los resultados del grupo de tendencias conocidas en los resultados de alguna filial. de los negocios y la situación de la sociedad, junto con una descripción de los principales riesgos e incertidumbres a los que se enfrenta".

Dada la relevancia de la información narrativa en el Reino Unido, el Accounting Standards Board (ASB), mediante guías de aplicación voluntaria, ha desarrollado el contenido mínimo del BR, denominado en su versión ampliada Operating and Financial Review ${ }^{9}$ (OFR). Desde abril de 2005 hasta enero de 2006, este informe llegó a ser obligatorio para todas las sociedades cotizadas. En línea con las guías de otros países, la guía del ASB solicitaba información no financiera y orientada hacia el futuro. Por último, el gobierno británico decidió no imponer requisitos adicionales a los de las Directivas Europeas y, por ello, el OFR volvió a convertirse en una guía voluntaria (Reporting Statement, RS1) que establece las mejores prácticas.

A partir de 2007, en el BR de las sociedades cotizadas, el legislador requiere nueva información prospectiva y no financiera: principales tendencias y factores de los que dependa el futuro de la entidad e información sobre medioambiente y personal apoyada en el uso de indicadores clave (Key Performance Indicators, KPI) no financieros (tabla 2). Desde entonces, esta información ha de ofrecerse "en la medida necesaria” para la comprensión de la evolución del negocio.

\footnotetext{
9 Operating and Financial Review, la primera guía voluntaria basada en las mejores prácticas disponibles sobre información narrativa del Accounting Standards Board, ASB, se elaboró en 1993 y fue revisada en 2003 y 2006. En 2013, el Financial Reporting Council (FRC) publicó el borrador de la nueva guía voluntaria que desarrolla el contenido del denominado Strategic Report.
} 


\section{Contenido del Strategic Report británico Objetivo}

El objetivo del Strategic Report es informar a los accionistas y ayudarles a valorar la gestión de los administradores en favor de la sociedad.

a. Una imagen fiel del negocio.

\section{Contenido}

b. Una descripción de los principales riesgos e incertidumbres que afronta la compañía.

El informe requiere un análisis equilibrado y completo sobre:

- La evolución y los resultados de la compañía durante el ejercicio.

- La situación del negocio al final del ejercicio.

Este análisis será consistente con la complejidad y tamaño del negocio.

El informe debe incluir, en la medida necesaria para comprender la evolución, gestión o situación del negocio:

- Un análisis con indicadores financieros clave de la gestión (Key Performance Indicators, KPI).

- Y cuando sea apropiado, la compañía proporcionará un análisis con otros KPI, incluyendo información relativa al medioambiente y los empleados (las sociedades medianas y pequeñas están exentas).

El informe, cuando sea apropiado, incluirá referencias y explicaciones adicionales de las cifras incluidas en las cuentas anuales de la compañía.

Nota: estos requisitos, que entraron en vigor en abril de 2005, se mantienen en la nueva regulación de 2013, para todas las compañías salvo las pequeñas ${ }^{1}$.

\section{Requisitos adicionales (sociedades cotizadas)}

En la medida necesaria para la comprensión de la evolución, resultados o posición del negocio:

a. Una descripción de la estrategia de la compañía.

b. Una descripción del modelo de negocio.

c. Los principales factores y tendencias que probablemente afectarán el futuro desarrollo, gestión y posición financiera del negocio de la compañía, e

d. Información sobre:

- asuntos medioambientales (incluyendo el impacto del negocio de la compañía sobre el entorno),

- los empleados de la compañía, y

- asuntos de la sociedad, la comunidad y los derechos humanos,

incluyendo información sobre cualquier política de la compañía en relación con esos asuntos, así como la efectividad de esas políticas.

Si el informe no contiene la información de los apartados a. b. y c., debe advertir qué clase de esas informaciones se han omitido.

e. Número de personas de cada sexo que sean administradores de la sociedad.

f. Número de personas de cada sexo que sean directivos de la sociedad.

g. Número de personas de cada sexo que sean empleados de la sociedad.

Nota: los requisitos (c) y (d) entraron en vigor en los ejercicios iniciados con posterioridad al 1 de octubre de 2007 y se han mantenido en las Regulaciones de 2013.

1. Los criterios para definir el tamaño son acordes con los establecidos por la Unión Europea.

Tabla 2. Contenido del Strategic Report británico

Fuente: Section 414C, Companies Act 2006 (Strategic Report and Director's Report) Regulations 2013

La presión para que las compañías incluyeran información prospectiva se relajó, mediante la incorporación de cláusulas de protección sobre la responsabilidad de los administradores, vigentes en la regulación actual (tabla 1). Así, la cláusula (12) de la sección 414C, Contenido del Informe estratégico, permite la omisión de información sobre proyectos en desarrollo inminente o contratos en curso de negociación que, a criterio de los administradores, pueda 
perjudicar los intereses de la compañía, y la cláusula complementaria 463 sobre el Informe estratégico y el Informe sobre remuneraciones establece que los administradores solo serán responsables ante la sociedad si sabían que la información era falsa o, en caso de omisión, si encubrieron informaciones deliberadamente. Por tanto, en ausencia de mala fe, no son responsables de los sucesos que prevean. De este modo, se fomenta la elaboración de informes más consistentes, en particular en lo relativo a la información previsional (Deloitte, 2007, pp. 2-3). Aunque el sistema de protección safe harbour se ha mantenido en la regulación actual, la seguridad relativa a la responsabilidad se cuestiona todavía (DBIS 2011, p. 26). Aproximadamente, el 45\% de las sociedades cotizadas británicas incluye en su Informe anual declaraciones de cautela (cautionary statement) sobre la información prospectiva (Deloitte, 2011).

Antes de la entrada en vigor de la regulación de 2006 y por tanto, de la protección safe harbour, cuando además se pensaba que el OFR tendría carácter obligatorio para las sociedades cotizadas, los miembros de organizaciones contables públicas y privadas recomendaban solicitar asesoramiento jurídico para elaborar sus propias cláusulas de protección sobre la información prospectiva del OFR (Independent Audit Limited, 2006, pp. 9-10; Mallett \& Slynn 2005, p. 36).

\subsubsection{Canadá}

En diciembre de 2007, el Canadian Securities Administrators, CSA, regulador canadiense de los mercados de valores, modificó la legislación sobre la información prospectiva vigente desde $2004^{10}$ (National Instrument, NI 51-102: Continuous Disclosure Obligations) que regula el MD\&A anual e intermedio (definición, principios y contenidos), y los demás informes que están obligadas a presentar las sociedades cotizadas (Annual Information Form y Business Acquisition Report).

La regulación canadiense del MD\&A fomenta que los administradores proporcionen información prospectiva, aunque no se considera obligatoria. Se requiere "una discusión de las tendencias conocidas e incertidumbres que con probabilidades razonables afectarán el futuro de la compañía". El MD\&A no exige previsiones detalladas de los ingresos o gastos futuros o de otros datos. La información prospectiva debe estar claramente identificada, para no ser confundida con la información histórica, por lo que se utilizará un lenguaje precautorio. Es crucial que el usuario comprenda las bases sobre las que se ha elaborado y se describan los factores materiales de riesgo que pueden hacer diferir los resultados previstos de los que se obtengan (hipótesis y riesgos asumidos). La norma añade que se debe actualizar aquella información prospectiva discutida en un MD\&A previo cuando, a la luz de los sucesos presentes o en ausencia de explicaciones adicionales, pueda inducir a errores. Se considera que una información es errónea cuando es excesivamente optimista o pesimista, carece de objetividad o no está adecuadamente explicada.

10 Los requisitos aplicables en los mercados de Canadá son los establecidos en el National Instrument 501-102 Continuous Disclosure Obligations por los reguladores regionales (British Columbia Securities Commission, Alberta Securities Commission, Ontario Securities Commission y Commission des valeurs mobilières du Québec), denominados colectivamente Canadian Securities Administrators (CSA). 


\section{A. Clasificación}

El CSA clasifica la información prospectiva en dos grupos: la información prospectiva general y la información prospectiva financiera. La primera es aquella relativa a posibles sucesos, condiciones o resultados de las operaciones, que está basada en hipótesis sobre futuras condiciones económicas y acciones en curso, incluyendo la información financiera prospectiva sobre los resultados, posición financiera o flujos de caja. Esta definición es muy amplia. No solo incluye los estados financieros previsionales sino cualquier información prospectiva de carácter no financiero, que contenga el MD\&A o cualquier otro informe presentado por escrito por una compañía, por ejemplo: revisiones estratégicas para el futuro, previsiones realizadas por una compañía sobre el crecimiento del sector o sobre los precios de las materias primas que adquiere habitualmente, indicadores clave de la gestión o ratios previstas, etc.

La segunda, información prospectiva financiera, en sus dos modalidades Financial Outlook y Future-Oriented Financial Information (FOFI), es la relativa a los resultados, posición financiera o flujos de caja con base en hipótesis sobre las condiciones económicas futuras y acciones en curso. La diferencia entre ambas modalidades es el formato: cuando se presenta en el formato de estados financieros históricos se denomina FOFI; si no adopta ese formato, se denomina Financial Outlook.

\section{B. Criterios}

Según el CSA, la información prospectiva se caracteriza, primero, por estar basada en unas hipótesis razonables y, segundo, por elaborarse siguiendo un método determinado. Para clasificar una información prospectiva como información prospectiva financiera, se exigen tres criterios adicionales:

- Ha de estar basada en hipótesis que deben ser razonables para las circunstancias concretas en las que se va a elaborar la información financiera prospectiva.

- Debe limitarse el período concreto para el que se estime (por lo general, no superior al año).

- Debe basarse en las mismas políticas contables que se espera que estén vigentes cuando se elabore la información contable histórica del período estimado.

\section{Requisitos}

En la tabla 3 se resumen los nuevos requisitos que se aplican en Canadá desde 2008 a toda la información prospectiva, tanto la publicada en el MD\&A, como en sitios web, anuncios de prensa o en medios publicitarios, siempre que no se emita de manera oral.

Como cabía esperar, todos los requisitos establecidos por el regulador se han incorporado a la última versión de la guía voluntaria canadiense sobre el MD\&A, en la que se explican con detalle (CA, 2009a, p. 45).

Dado que, por definición, la información prospectiva es menos fiable que la información histórica, es lógico que el regulador exija que se identifique como tal, que se expliquen las bases sobre las que se ha desarrollado, las variaciones que pueden surgir entre los resultados reales y previstos y los factores que las han originado. Estos factores serán originales para cada empresa en cada momento y específicos en función 


\begin{tabular}{ll}
\hline & Requisitos para toda la información prospectiva \\
\hline Generales & Identificación de este tipo de información como tal. \\
& $\begin{array}{l}\text { Advertencia a los usuarios de que los resultados reales pueden diferir de los prospectivos. } \\
\text { Factores materiales de riesgo que hacen que los resultados reales puedan diferir de los previstos. } \\
\text { Factores materiales e hipótesis usados para desarrollar la información prospectiva. }\end{array}$ \\
\hline Actualización & $\begin{array}{l}\text { Sucesos o circunstancias del período que con probabilidades razonables han hecho que los resultados } \\
\text { actuales difieran materialmente de los previstos en la información prospectiva previamente emitida. }\end{array}$ \\
& $\begin{array}{l}\text { Diferencias esperadas. } \\
\text { Descripción de la política de actualización de la información prospectiva. }\end{array}$ \\
\hline Retirada de & $\begin{array}{l}\text { Discusión de cualquier decisión de retirar información prospectiva previamente emitida. Sucesos y } \\
\text { circunstancias que han provocado tal decisión, por cuanto han causado que la información deje de ser válida. }\end{array}$ \\
información & Requisitos adicionales para la información prospectiva financiera \\
\hline Propósito con el que se elabora la información prospectiva, con la advertencia de que puede no ser válida para otros \\
propósitos.
\end{tabular} $\begin{aligned} & \text { La fecha en que los gestores aprueban la información, si el documento que contiene esa información no está fechado. } \\
& \text { Discusión de las diferencias materiales entre los resultados actuales, intermedios o anuales, y los previstos en la } \\
& \text { información financiera previamente emitida. }\end{aligned}$

Tabla 3. Requisitos de la información prospectiva en Canadá (Forward Looking Information, FLI)

Fuente: National Instrument (NI 51-102F) Continuous Disclosure Obligations, apartados 4A, 4B y 5.8. Requisitos vigentes desde el ejercicio 2008

del contenido o información prospectiva tratada.

Puesto que la información prospectiva puede figurar en páginas web, en notas de prensa, etc., los administradores deben tener en cuenta los cambios que ya se hayan comunicado antes de elaborar el MD\&A (CA, 2008a, p. 5, p. 9).

Muchas empresas prefieren ofrecer la información prospectiva en forma de rango o intervalo de resultados posibles en lugar de lanzar una sola estimación concreta de los resultados. Cuando sea posible, es recomendable utilizar un análisis de la sensibilidad de la información cuantitativa a los cambios en las hipótesis que se barajan. Otra posibilidad es manejar, por ejemplo, dos escenarios diferentes según se lleven a cabo dos acciones alternativas, que tendrán como resultado dos estados financieros prospectivos distintos (CA, 2008a, p. 4, p. 6).

En cumplimiento de su responsabilidad de aprobar el MD\&A, el Comité de Auditoría debe revisar la información prospectiva del informe. El Consejo debe valorar la razonabilidad de las hipótesis asumidas y revisar los sistemas y procesos por los que se genera (CA, 2008a, p. 7).

Por último, la regulación de los mercados secundarios prevé cláusulas de protección safe harbour sobre la información prospectiva del MD\&A, pero solamente tienen validez si se cumplen las condiciones que figuran en la tabla 1. Por este motivo y dada la complejidad de la legislación sobre responsabilidad civil en los mercados canadienses, el CA recomienda a los administradores solicitar asesoramiento jurídico al elaborar la información prospectiva (CA, 2008b, p. 4). En el caso del Reino Unido no se especifican condiciones, sino que la cláusula safe harbour se aplica a toda la información prospectiva, siempre que figure en el Strategic Report o en el Informe de Remuneraciones. 


\section{Análisis de la evidencia empírica}

\subsection{Objetivo y metodología}

A fin de encontrar indicios que demuestren si los beneficios y limitaciones que la teoría asocia a la información prospectiva se cumplen en la práctica empresarial, hemos realizado una revisión de la evidencia empírica publicada en revistas científicas de Contabilidad.

En primer lugar se llevó a cabo una búsqueda en la base de datos Web of Science (WOS), introduciendo las palabras clave: MD\&A, OFR, voluntary disclosure, prospective financial information, forward-looking diclosures y narratives. De los trabajos encontrados (más de 100) se seleccionó un total de 38 trabajos empíricos, esencialmente artículos ${ }^{11}$, que tratan la información narrativa prospectiva contenida en los informes anuales de sociedades cotizadas. Los criterios de selección fueron los siguientes:

1. Los trabajos aplican alguna de las metodologías delimitadas por Vivien Beattie, Bill McInnes y Stella Fearnley (2004) en su clasificación de estudios empíricos sobre la calidad de la información narrativa, descritas en la figura 1:

a. Ratings basados en las puntuaciones de los analistas — de ahí su carácter subjetivo-de la Financial Analysts Federation (FAF) de la Association for Investment Management

11 A excepción de Volkan Muslu, Suresh Radhakrishnan, K. R. Subramanyam y Dongkuk Lim (2011), los restantes trabajos han sido publicados en revistas científicas específicamente dedicadas a la divulgación de la investigación en Contabilidad.
Research (AIMR) ${ }^{12}$. Estas puntuaciones representan una medida global de toda la información que publican las empresas de diferentes sectores, que pondera tres dimensiones: información anual (estados financieros e información narrativa, incluido el MD\&A), información trimestral e información relativa a "las relaciones con inversores". En esta categoría, los analistas valoran cuán accesible es la dirección de la empresa para los analistas y la frecuencia e interés de los gestores en sus presentaciones y entrevistas.

b. Análisis del contenido temático (thematic content analysis). Es un método de investigación reconocido en las ciencias sociales que permite identificar los contenidos principales de un texto al establecer inferencias sistemáticas sobre las categorías que se observan. A diferencia de los estudios del contenido basados en índices, el investigador no parte de unos contenidos especificados ex ante cuya aparición y calidad se pretenden medir, sino que los textos se analizan, precisamente, para encontrar los contenidos relevantes.

c. Análisis sintáctico o de legibilidad (syntactic analysis; readability studies). Esta metodología suele utilizarse para probar la hipótesis de impression management, también conocida como ofuscación (readability obfuscation). Consiste en utilizar la complejidad de la narrativa, medida por un índice de legibilidad, para ocultar malos resultados. Estos estudios

12 Desde 2004, su nombre es CFA Institute. Estos ratings sobre la calidad de la información solo estuvieron disponibles en Estados Unidos hasta 1997. Su gran ventaja fue que permitieron análisis estadísticos a gran escala, siguiendo la tradición de la investigación en Estados Unidos, que favorece los trabajos cuantitativos (investigación positiva). 


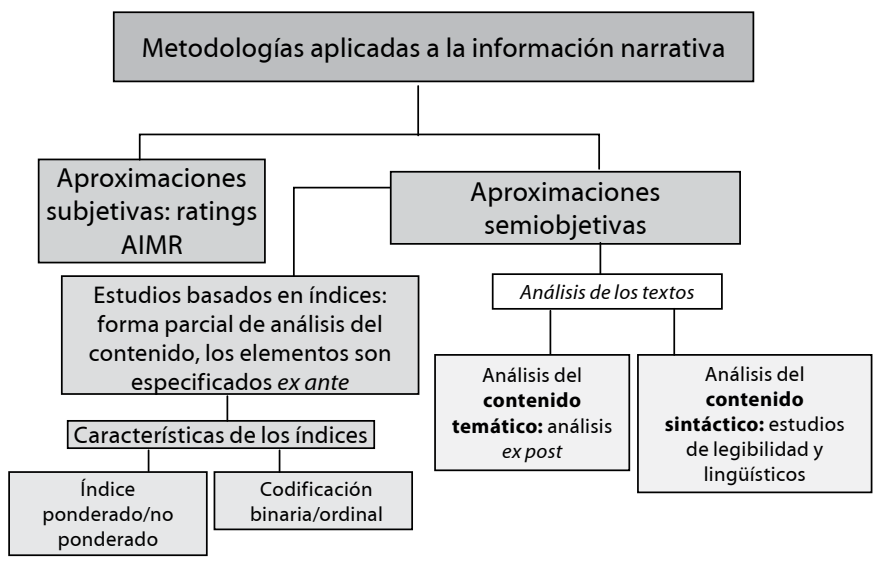

Figura 1. Metodologíasaplicadas a la información narrativa Fuente: Vivien Beattie, Bill McInnes y Stella Fearnley (2004)

estiman la complejidad de los textos utilizando las denominadas fórmulas de legibilidad —que miden la longitud de las palabras y de las oraciones-o identificando otras variables relacionadas con la dificultad del texto. Una vez medido el nivel de complejidad de un informe, la medida obtenida se relaciona con otras variables, la gestión realizada, frecuentemente, $\mathrm{u}$ otras representativas de características de la empresa como tamaño, riesgo o sector (Courtis, 1998, p. 461).

d. Índices de revelación. El índice es un indicador numérico formado por variables categóricas que toman, normalmente, el valor uno (1) si la empresa divulga el elemento informativo, o cero (0) en caso contrario. Los investigadores asumen en sus estudios de contenido basados en índices que la cantidad de información ofrecida sobre determinados elementos constituye una aproximación de la calidad, es decir, la cantidad y la calidad de la información están positivamente relacionadas (Botosan, 1997, p. 324).
En la tabla 4 se muestra el número de estudios analizado por cada una de las metodologías propuestas por Vivien Beattie, Bill McInnes y Stella Fearnley (2004):

\begin{tabular}{lc}
\hline \multicolumn{2}{c}{$\begin{array}{c}\text { Clasificación de los trabajos según la } \\
\text { metodología empleada }\end{array}$} \\
\hline Estudios basados en ratings de analistas financieros & 4 \\
\hline Análisis del contenido temático & 17 \\
\hline Análisis de contenido sintáctico & 6 \\
\hline Estudios del contenido basados en índices & 11 \\
\hline Total & 38 \\
\hline
\end{tabular}

Tabla 4. Clasificación de los trabajos según la metodología empleada

Fuente: elaboración propia

2. Han sido publicados después de 1994, puesto que Michael Jones y Paul Shoemaker revisaron los análisis de contenido publicados hasta esa fecha, en sus dos vertientes: temática (36 estudios) y sintáctica (32 estudios). En su trabajo, explican la metodología de este 
tipo de análisis, los clasifican según su finalidad y comentan los resultados alcanzados.

3. Que la "materia prima" u objeto de análisis del estudio sea la denominada información narrativa o voluntaria, esto es, la contenida en el Informe anual excluidos los estados contables, bien en su totalidad, bien en alguna de sus secciones: el informe de gestión y la carta del presidente preferentemente. En algunos casos, los investigadores no especifican si la información que han analizado es la que se encuentra en esas secciones en particular; simplemente se refieren a la información prospectiva encontrada "en la información narrativa del Informe anual". Por tanto, se excluyeron aquellos trabajos que, aun utilizando las técnicas del análisis de contenido o los índices, se han aplicado a otro tipo de información, en concreto: información social o medioambiental, responsabilidad social corporativa, información sobre el capital intelectual o humano, informes de previsiones de analistas financieros, folletos de emisiones y anuncios de resultados de los gestores. El número de estudios según la información analizada, se muestra en la tabla 5:

4. Se han seleccionado prioritariamente aquellos trabajos publicados en el ranking de las revistas que Beattie (2005, pp. 89-90) identifica como representativas de las "élites" de la investigación británica y estadounidense. Como se observa en la tabla 6, el 61\% de los trabajos analizados se ha obtenido de las mismas.

Una vez seleccionados, los estudios se han clasificado atendiendo a su finalidad. Como resultado, hemos identificado dos grupos: pri- mero, estudios que demuestran la utilidad y/o limitaciones de la información prospectiva. Estos estudios se citan en el apartado 4.2. Segundo, estudios que definen las características de las empresas más proclives a divulgar este tipo de información (apartado 4.3).

\begin{tabular}{ll}
\hline \multicolumn{2}{c}{ Clasificación de los trabajos } \\
\hline Ratings AIMR o equivalentes & 2 \\
\hline $\begin{array}{l}\text { Información narrativa o voluntaria contenida en } \\
\text { el Informe anual }\end{array}$ & 18 \\
\hline Informe de gestión & 14 \\
\hline Carta del presidente & 4 \\
\hline Total & 38 \\
\hline
\end{tabular}

Tabla 5. Clasificación de los trabajos atendiendo a la información objeto de análisis Fuente: elaboración propia

\begin{tabular}{lrl}
\hline \multicolumn{1}{c}{ Número de trabajos } & Origen \\
obtenidos de cada revista & Oring \\
\hline $\begin{array}{l}\text { 1. Accounting, Auditing \& } \\
\text { Accountability Journal }\end{array}$ & 4 & Reino Unido \\
\hline $\begin{array}{l}\text { 2. Accounting and Business } \\
\text { Research }\end{array}$ & 6 & Reino Unido \\
\hline $\begin{array}{l}\text { 3. Contemporary Accounting } \\
\text { Research }\end{array}$ & 3 & Canadá \\
\hline $\begin{array}{l}\text { 4. The Accounting Review } \\
\text { 5. Journal of Accounting }\end{array}$ & 1 & Estados Unidos \\
\hline Research & 3 & Estados Unidos \\
\hline 6. Journal of Business Finance & & \\
\& Accounting & 1 & Reino Unido \\
\hline 7. The British Accounting & & \\
Review & 2 & Reino Unido \\
\hline 8. The European Accounting & & \\
Review & 2 & Bélgica \\
\hline 9. Journal of Accounting and & & \\
Economics & 1 & Estados Unidos \\
\hline Total en estas revistas & 23 & $61 \%$ \\
\hline Otros artículos & 15 & $39 \%$ \\
\hline \begin{tabular}{l} 
Total trabajos analizados \\
\hline
\end{tabular} & $100 \%$ \\
\hline
\end{tabular}

Tabla 6. Fuentes

Fuente: elaboración propia 


\subsection{Evidencia empírica sobre la}

\section{utilidad y limitaciones de la información prospectiva}

Este apartado recoge las principales aportaciones de los estudios analizados que constatan el valor predictivo o utilidad de la información prospectiva.

\subsubsection{Investigaciones relativas a la} incidencia en el valor de mercado de las empresas de la información prospectiva sobre el beneficio, basadas en el modelo

\section{de Collins}

Para validar esta hipótesis, esto es, si la información prospectiva influye sobre el valor de mercado de las empresas, diversos autores han aplicado el modelo de Daniel W. Collins, S. P. Kothari, Jay Shanken y Richard G. Sloan (1994). Estos autores revisan la literatura previa sobre la relación entre cotizaciones y bene- ficios actuales, y concluyen que la correlación entre estas variables es baja, debido a que los criterios de reconocimiento del modelo contable impiden contabilizar determinados flujos de caja esperados asociados a gastos presentes que se difieren y son reflejados en los beneficios de períodos futuros. Este efecto se denomina ausencia de oportunidad del resultado para captar sucesos relevantes (lack of timeliness) (Collins, Kothari, Shanken \& Sloan, 1994, p. 292).

Según el modelo de Collins, cuyo detalle aparece en la tabla 7, la rentabilidad actual de las acciones no solo depende del crecimiento del beneficio del período presente - puesto que el mercado tiene acceso a información relevante diferente al beneficio-, sino que también resulta afectado por los beneficios futuros, que se estiman por medio de la información narrativa, prospectiva en particular.

\title{
Modelo de Collins, Kothari, Shanken \& Sloan (1994)
}

\begin{abstract}
El modelo de regresión de Daniel W. Collins, S. P. Kothari, Jay Shanken y Richard G. Sloan, denominado price earnings lead lag, explica el cambio del valor de la rentabilidad presente de las acciones $\left(\mathrm{R}_{\mathrm{t}}\right)$ en función de dos variables fundamentales: el crecimiento del beneficio actual o beneficio no anticipado $\left(\mathrm{X}_{\mathrm{t}}\right)$ y los cambios en los beneficios futuros $\left(\mathrm{X}_{\mathrm{t}+1}, \mathrm{X}_{\mathrm{t}+2}, \mathrm{X}_{\mathrm{t}+3}\right)($ anticipados o esperados) de los próximos de tres años. En la medida en que la rentabilidad actual refleje información sobre beneficios futuros (obtenida en $t$ y representada por $\mathrm{X}_{\mathrm{t}+1}, \mathrm{X}_{\mathrm{t}+2}, \mathrm{X}_{\mathrm{t}+3}$ ) mayores serán los coeficientes de respuesta $\mathrm{b}_{\mathrm{k}}$.
\end{abstract}

$R_{t}=b_{0}+b_{1} X_{t}+\sum_{k=1}^{3} b_{k+1} X_{t+k}+\sum_{k=1}^{3} b_{k+4} R_{t+k}+b_{8} E P_{t-1}+b_{9} A G_{t}+e_{t}$

Además, se incorporan al modelo otras variables de control, para corregir problemas de medida del error estadístico:

1. La parte del cambio en el beneficio actual que podría haber sido anticipada en un período previo, o previsión del mercado sobre el crecimiento del beneficio actual, se estima mediante dos variables:

- La ratio $\mathrm{E} / \mathrm{P}_{\mathrm{t}-1}$, earnings over price (beneficio sobre precio de mercado) que representa la previsión del mercado del crecimiento del beneficio, calculado en $t-1$ sobre el precio o cotización al inicio del período.

- El crecimiento del activo, $A G_{t}$ (u otros indicadores del incremento en la capacidad productiva o la inversión).

2. Las noticias sobre beneficios futuros recibidas en los períodos posteriores a $t$, quedan reflejadas por las cotizaciones futuras: $R_{t+k}$ (de tres períodos: $t+1, t+2$ y $t+3$ ) que representan los cambios no esperados en los beneficios futuros (no correlacionadas con las cotizaciones actuales, variable dependiente; sí con los beneficios futuros). Esta variable refleja el crecimiento de los beneficios futuros no anticipados en el período $t$, sino posteriormente. 
Los estudios revisados que han aplicado el modelo de Collins, Kothari, Shanken y Sloan a la información narrativa, coinciden en que el beneficio actual no explica suficientemente el valor presente de la rentabilidad de las acciones y que la información prospectiva sobre los beneficios guarda relación con las cotizaciones presentes.

Los primeros estudios que aplicaron el modelo de Collins, Kothari, Shanken y Sloan, utilizaron como medida de la variable "nivel informativo" los ratings estadounidenses de analistas financieros miembros de la AIMR, que representan una medida global de la calidad de la información publicada por las empresas más representativas de cada sector. Estos estudios llegan a la conclusión de que las empresas mejor calificadas, es decir, aquellas que ofrecen mayor información sobre beneficios futuros, permiten que el proceso de formación de los precios se haga de una manera más eficiente (Gelb \& Zarowin, 2002; Lundholm \& Myers, 2002).

Posteriormente, en ausencia de los ratings AIMR, investigadores británicos han elaborado sus propios índices para medir el nivel de información divulgada e introducirlo en el modelo de Collins, Kothari, Shanken y Sloan. Thomas Schleicher y Martin Walker (1999) elaboraron tres índices de calidad de la información narrativa del informe de gestión (OFR): sobre el informe en su conjunto, sobre la información prospectiva y sobre la información segmentada. A partir de una muestra de empresas británicas cotizadas lograron demostrar que la información voluntaria del OFR, en especial la información prospectiva, es útil para que el mercado des- cuente en las cotizaciones los cambios esperados en los beneficios futuros, en concreto, del período siguiente y subsiguiente al ejercicio en que se explican las cotizaciones (Schleicher \& Walker, 1999, p. 333-334).

Khaled Hussainey, Thomas Schleicher y Martin Walker (2003) realizaron un análisis de contenido sobre la información voluntaria a gran escala (unas ochocientas empresas). Los autores obtienen dos tipos de puntuaciones: las relativas a la calidad de la información voluntaria en general y las relativas a la información voluntaria de carácter prospectivo, y constatan que la información prospectiva sobre los beneficios ayuda al mercado a anticipar con mayor precisión los futuros cambios en los beneficios de las empresas (Hussainey, Schleicher \& Walker, 2003, p. 276). La ampliación posterior de este trabajo a una muestra mayor de empresas (dos mil quinientas aproximadamente) confirma la relación entre las cotizaciones actuales y la información de carácter prospectivo sobre los beneficios en dos tipos de empresas: empresas con beneficios y con pérdidas. La asociación es significativamente mayor en estas últimas, en las que los resultados presentes no son un buen indicador de la capacidad de generar ingresos a largo plazo (Schleicher, Hussainey \& Walker, 2007, p. 170).

Jesper Banghøj y Thomas Plenborg (2008) confirman la relación entre los beneficios actuales y futuros y la rentabilidad actual de las acciones, pero no encuentran que la capacidad explicativa del modelo mejore al introducir como variable explicativa el nivel informativo, medido por un índice sobre información voluntaria contenida en el Informe anual, inspirado 
en el Informe Jenkins (1994), en otros informes y en la literatura previa, incluso cuando los autores seleccionan en el índice los indicadores relacionados con beneficios futuros. Algunas causas pueden justificarlo: el tamaño de la muestra es reducido y se limita al sector industrial; las empresas danesas se caracterizan por una alta concentración de la propiedad, por ofrecer escasa información voluntaria o ser esta poco relevante para los inversores puesto que obtienen la información por otros canales (Banghøj \& Plenborg, 2008, p. 161).

Junto al nivel de información prospectiva revelado, Khaled Hussainey y Martin Walker (2009, p. 37) advierten que la política de dividendos es otra variable de comunicación financiera que da señales al mercado sobre los beneficios esperados. Los autores demuestran que la propensión a repartir dividendos y la información voluntaria mejoran la capacidad del mercado para anticipar cambios en los beneficios futuros, en las empresas con alto crecimiento, no así en las de bajo crecimiento. Estos resultados sugieren que, hasta cierto punto, aquellas variables son formas sustitutivas de comunicación.

Volkan Muslu, Suresh Radhakrishnan, K. R. Subramanyam y Dongkuk Lim (2011, p. 22, p. 24; 2014, p. 17) comprueban que la información prospectiva del MD\&A tiene un impacto especial sobre la rentabilidad presente de las acciones en el caso de las empresas que se caracterizan por desenvolverse en un entorno informativo rico (alto seguimiento de analistas financieros), en el de aquellas cuyos beneficios experimentan disminuciones importantes respecto al ejercicio anterior o respecto a las previsiones sobre el beneficio de analistas y, en general, en aquellas en las que las cotizaciones actuales no guardan una estrecha correlación con los beneficios futuros.

\subsubsection{Otras investigaciones que} analizan la utilidad de la información prospectiva para el mercado y para los analistas financieros

Otros investigadores demuestran la utilidad de la información prospectiva al comprobar el cumplimiento de las previsiones anunciadas por los gestores. A tal efecto, suelen verificar la asociación entre el tono de las previsiones positivo o negativo-, y la evolución de la gestión posterior de la entidad, representada por determinadas variables financieras - cotizaciones y gestión realizada, habitualmente- . Por otra parte, la validez de la información prospectiva también se ha contrastado al analizar su capacidad para incrementar la precisión de las previsiones de analistas financieros.

Stephen H. Bryan (1997, pp. 297-298) señala las relaciones que hay entre la información prospectiva del MD\&A y los cambios en determinadas variables financieras de las empresas durante los tres primeros ejercicios posteriores a la publicación de los informes (por ejemplo, entre las inversiones planificadas y las cotizaciones). A. Eliane Eikner, Hassan Hefzi y G. William Glezen (2000) encuentran una asociación positiva entre un índice que mide el signo de la información prospectiva (previsiones positivas o negativas) y la rentabilidad de las acciones. También Feng Li (2010, p. 1050), en un análisis del contenido a gran escala (ciento cuarenta mil observaciones) sobre la informa- 
ción prospectiva de los MD\&A incluidos en los informes anuales y trimestrales presentados a la SEC, demuestra que el tono (positivo o negativo) de las previsiones está asociado positivamente con la gestión realizada y futura, así como a otras variables como el crecimiento (ratio book to market) o la edad de la empresa, y negativamente respecto a la volatilidad de las acciones y el tamaño.

Khaled Hussainey y Jinan Aal-Eisa se centran en empresas en declive, es decir, aquellas que experimentan un descenso en los beneficios tras un crecimiento sostenido. Comparan la política de dividendos y la divulgación de información prospectiva como canales alternativos para anunciar una mejora en los beneficios esperados. En el período de la caída de los beneficios, identifican los cambios en la política de dividendos y en la emisión de información prospectiva de una muestra de empresas cotizadas británicas, lo que demuestra que solamente la segunda variable está relacionada con incrementos de los beneficios en el futuro (Hussainey \& Aal-Eisa, 2009, p. 452).

Los investigadores también han estudiado la utilidad de la información prospectiva para los analistas financieros. En una encuesta realizada por Peter M. Clarkson, Jennifer L. Kao y Gordon D. Richardson (1999), estos analistas destacan el valor de la información prospectiva publicada en el MD\&A (planes de inversiones o previsiones por segmentos, por ejemplo). Consideran que este informe es útil para el análisis financiero porque da acceso a información detallada y actual en un informe único y porque confirma las informaciones previas emitidas por los gestores (Clarkson, Kao \& Richardson, 1999, p. 121).

Orie E. Barron, Charles O. Kile y Terrence B. O'Keefe (1999, p. 98) miden la asociación entre la calidad de los MD\&A, valorados por los ratings de la SEC y los errores en las previsiones sobre los beneficios de esas empresas realizadas por los analistas financieros del Institutional Brokers' Estimate System (I/B/E/S). Estos autores encuentran que altos niveles en los ratings están asociados con bajos errores y dispersión en las previsiones de los beneficios, y opinan, al igual que la SEC, que la información prospectiva de los MD\&A de alta calidad es relevante para predecir los beneficios.

Así mismo, los estudios basados en índices sobre empresas europeas realizados por Raf Orens y Nadine Lybaert (2007, p. 265) o Saverio Bozzolan, Marco Trombetta y Sergio Beretta (2009, p. 464) demuestran la asociación existente entre la cantidad de información prospectiva - cuantitativa, en este último estudio-y la precisión de las previsiones de analistas financieros.

Por su parte, Volkan Muslu, Suresh Radhakrishnan, K. R. Subramanyam y Dongkuk Lim (2011, 2014) consideran que el MD\&A es un vehículo de comunicación complementario a la información que elaboran los analistas financieros, puesto que información prospectiva y seguimiento por parte de analistas financieros presentan una asociación positiva. 


\subsubsection{Sobre la escasa información prospectiva emitida y el sesgo en las previsiones}

Los estudios empíricos sobre el MD\&A/OFR de empresas cotizadas en Estados Unidos, Reino Unido, Canadá o Australia revelan que con frecuencia los análisis prospectivos son deficitarios y sesgados (Clarkson, Kao \& Richardson, 1994; Schleicher, 2012; Sydserff \& Weetman, 1999; Yeoh 2010).

La mayoría ofrece exclusivamente información cualitativa (a favor, Bryan, 1997, p. 289; Schleicher \& Walker 1999, p. 324) y se aprecia un claro sesgo hacia las previsiones optimistas o neutrales (Kent \& Ung, 2003; O'Sullivan, Percy \& Stewart, 2008). La investigación llevada a cabo por Suzan Abed, Saleh Al-Okdeh y Khalil Nimer (2011) también pone de manifiesto la escasa información prospectiva publicada por las empresas cotizadas, en este caso en el Amman Stock Exchange (ASE) jordano. En el mismo sentido apuntan los trabajos de Muslu, Radhakrishnan, Subramanyam y Lim (2011 y 2014) en los que analizan la información prospectiva de casi cinco mil sociedades cotizadas estadounidenses: el porcentaje de frases de carácter prospectivo en el MD\&A se sitúa en el 12,6\%.

Thomas Schleicher y Martin Walker (2010) estudian si la información prospectiva contenida en el Informe anual está sesgada. Aplican el análisis de contenido temático a una amplia muestra de empresas cotizadas británicas y alcanzan dos conclusiones: primera, las empresas que esperan grandes disminuciones en sus ingresos o en su margen de explotación, utilizan un tono positivo en la información prospectiva para mejorar la imagen de la entidad; segunda, las empresas con pérdidas, las que presentan un perfil de mayor riesgo (variabilidad de la rentabilidad) y las que son seguidas por analistas financieros, tienden a informar con un tono positivo u optimista (Schleicher \& Walker 2010, p. 388).

Estas conclusiones son consistentes con las de otros estudios previos que trabajan la hipótesis de impression management, aplicada tradicionalmente a la información narrativa histórica. Aquel término está asociado en la literatura contable a algunos métodos informativos como el uso de gráficos e imágenes y, en general, a la información narrativa (Sydserff \& Weetman 2002, p. 525-526). Esta hipótesis prueba si los gestores emplean determinadas estrategias al elaborar los informes, como utilizar un lenguaje complejo o presentar reiteradamente los mismos contenidos, explicaciones o actitudes asociados a determinadas circunstancias, normalmente, a la gestión - positiva o negativa-de la empresa. Esto significa que ante una gestión deficiente, los directivos dificultan el lenguaje de los informes o adoptan un comportamiento optimista, al tiempo que justifican sus resultados con causas externas. Por lo general, estos estudios confirman total o parcialmente la presencia de estrategias de impression management (Aerts, 2001, Balata \& Breton, 2005; Clatworthy \& Jones, 2003, 2006; Courtis, 1998, 2004; Li, 2008; Sydserff \& Weetman, 2002).

Schleicher (2012) no solo demuestra la relación negativa entre la gestión realizada y el tono sesgado de la información prospectiva, sino que identifica otros atributos o estrategias asociados a las previsiones. En el caso de que la gestión sea negativa (descenso en las ventas o 
beneficios), las previsiones tienden a circunscribirse a segmentos específicos, se condicionan a determinados sucesos y se refieren a objetivos generales; pero si la gestión es positiva, las previsiones se suelen establecer sobre un horizonte temporal de corto plazo, se refieren a datos o ratios concretos y se comparan con los resultados de años anteriores.

\subsection{Evidencia empírica sobre las características de las empresas que publican más información prospectiva}

El análisis de las características que presentan aquellas empresas que divulgan mayor cantidad de información prospectiva requiere la construcción de un índice que cuantifique esta variable. El principal inconveniente de la metodología de los índices de revelación es la subjetividad en su diseño: selección de la información y sistema de puntuación. Dado que la construcción de los índices puede determinar los resultados de las mediciones, las conclusiones de estos estudios han de tomarse con cautela (Bravo-Urquiza, Abad-Navarro \& Trombetta, 2009, p. 268).

En su estudio, Peter M. Clarkson, Jennifer L. Kao y Gordon D. Richardson (1994, p. 435445) demuestran que las empresas de mayor tamaño son más propensas a fijar previsiones, que unos sectores son más proclives que otros a establecer predicciones, que hacer previsiones en un ejercicio es independiente de volver a efectuarlas en el ejercicio siguiente y que tanto las previsiones positivas como las negativas se cumplen mayoritariamente (en un 64 y $98 \%$, respectivamente). Así mismo, identifican tres variables relacionadas con la divulgación de previsiones: la gestión financiera, las necesidades de financiación y la llegada de competidores al mercado. Concretamente, las empresas con buenos resultados son más propensas a hacer previsiones y a solicitar financiación, pero esta propensión a fijar previsiones disminuye ante la presencia de un competidor. En un estudio posterior sobre la calidad del MD\&A y sus componentes, relacionan los incrementos en el nivel de información prospectiva con la gestión realizada (Clarkson, Kao \& Richardson, 1999, p. 130).

Suzan Abed, Saleh Al-Okdeh y Khalil Nimer (2011) encontraron que el nivel de información prospectiva está relacionado positivamente con el tamaño de la sociedad (a favor, Flöstrand \& Ström, 2006; Hossain, Ahmed \& Godfrey, 2005; Kent \& Ung, 2003; Muslu, Radhakrishnan, Subramanyam \& Lim, 2014; O'Sullivan, Percy \& Stewart, 2008) y negativamente con el nivel de competencia (a mayores barreras de entrada, menor nivel de información prospectiva) (a favor, Hossain, Ahmed \& Godfrey, 2005). Sin embargo, sus resultados muestran una asociación negativa entre la gestión realizada y la información prospectiva, lo que supone que esta se utiliza para explicar el resultado contable cuando es negativo (a favor, Muslu, Radhakrishnan, Subramanyam \& Lim, 2014). Este argumento también queda sustentado por Khaled Aljifri y Khaled Hussainey (2007) en su estudio sobre información prospectiva de empresas cotizadas en los Emiratos Árabes, en el que la rentabilidad y el endeudamiento son variables relacionadas negativamente, en el primer caso, y positivamente, en el segundo, con un índice previamente calculado que mide la cantidad de información prospec- 
tiva (a favor, Hossain, Ahmed \& Godfrey, 2005; O'Sullivan, Percy \& Stewart, 2008).

En relación con la característica de alto crecimiento o capacidad de crecimiento de las compañías Suzan Abed, Saleh Al-Okdeh y Khalil Nimer (2011), Mahmud Hossain, Kamran Ahmed y Jayne M. Godfrey (2005), Volkan Muslu, Suresh Radhakrishnan, K. R. Subramanyam y Dongkuk Lim (2014) y Madonna O'Sullivan, Majella Percy y Jenny Stewart (2008) descubren una relación positiva en relación con la información prospectiva. En un estudio sobre la cantidad de información prospectiva publicada en el Informe anual de doscientas empresas del S\&P500, Per Flöstrand y Niklas Ström (2006, p. 590) comprobaron que está relacionada con el nivel de intangibles que muestran los balances.

Por último, otros autores han relacionado la divulgación de información prospectiva con los mecanismos que garantizan el buen gobierno corporativo. O'Sullivan, Percy y Stewart (2008, p. 30) encontraron una relación positiva entre la información prospectiva emitida en el informe anual y la calidad del sistema de gobierno corporativo, en concreto, de la auditoría (en contra, Abed, Al-Okdeh \& Nimer, 2011; Aljifri \& Hussainey, 2007). Así mismo, María Cristina Abad-Navarro y Francisco Bravo-Urquiza (2012, p. 21) demostraron que la cantidad de información previsional divulgada en los informes anuales está relacionada con la efectividad del Consejo de administración.

\section{Conclusiones}

La revisión de la normativa internacional realizada en este trabajo nos ha permitido consta- tar la naturaleza pseudovoluntaria y subjetiva de la información financiera prospectiva, y su carácter esencialmente cualitativo. Hemos constatado, así mismo, la tendencia de los organismos reguladores internacionales (CSA, 2009; CNMV, 2013; FRC, 2013; IASB, 2010) a normalizar este tipo de información mediante la emisión de guías para la elaboración del informe de gestión. Estas guías, de adhesión voluntaria, incorporan un conjunto de principios de transparencia metodológica diseñados para garantizar la fiabilidad de la información prospectiva (como identificación, explicación de las hipótesis sobre las que se construye, factores materiales de riesgo que afectan las previsiones y discusión de las desviaciones).

A fin de mejorar la comparabilidad de esta información sugerimos, en el caso de las sociedades cotizadas, la posibilidad de incorporar estos principios a la regulación de los países que han adoptado las normas del International Accounting Standards Board (IASB), en la medida en que, como primer paso, estas sociedades vayan progresivamente adoptando los estándares voluntarios en su praxis informativa.

A pesar de la reconocida relevancia de la información prospectiva para los inversores, su desarrollo aún es incipiente, debido a los inconvenientes que presenta su difusión: pérdida potencial de competitividad y responsabilidad de los administradores de la compañía por el incumplimiento de sus previsiones. En los tres casos analizados (Estados Unidos, Reino Unido y Canadá) se consolida el sistema de cláusulas de protección - exenciones de responsabilidad civil—de los administradores sobre la información emitida (safe harbour provisions), como 
el mecanismo adecuado para evitar las reticencias de los consejos a la hora de publicar este tipo de información y fomentar la flexibilidad e innovación en su presentación.

La revisión posterior de la evidencia empírica pone de manifiesto la tendencia actual de aplicar a la información narrativa exclusivamente de carácter prospectivo, las dos metodologías utilizadas tradicionalmente en la investigación contable sobre información narrativa: los índices de revelación y el análisis del contenido (temático y sintáctico).

Este análisis nos ha permitido clasificar los estudios en dos grupos, atendiendo a su finalidad. El primero está formado por aquellos análisis del contenido encaminados a demostrar la utilidad y las limitaciones de la información prospectiva. La literatura da cuenta de los beneficios de la información prospectiva, entre otros: su impacto sobre las cotizaciones presentes - en particular en empresas con pérdidas y en empresas con alto crecimiento-, su capacidad para reducir el error y la dispersión de las previsiones sobre el beneficio de analistas financieros y su utilidad en términos de cumplimiento de las previsiones anunciadas. No obstante, buena parte de los análisis de contenido revisados confirman total o parcialmente la presencia de estrategias diseñadas para sesgar la información prospectiva (hipótesis de impression management).

El segundo grupo de estudios identificado se ocupa de la delimitación de los factores relacionados con el nivel de información prospectiva publicada. Los investigadores están diseñando índices de revelación adaptados a este tipo de información en particular, dado que las variables asociadas a la información prospectiva pueden diferir de las asociadas a la información voluntaria en general. Hay relativo consenso sobre los factores asociados con el nivel de información prospectiva, positiva o negativamente (tamaño, alto crecimiento y calidad de los sistemas de gobierno corporativo en el primer caso; nivel de competencia en el segundo). Sin embargo, respecto a la gestión realizada los resultados son mixtos, lo cual sugiere la necesidad de continuar investigando sobre si la información prospectiva constituye un vehículo de información complementario o alternativo a la cifra del beneficio.

\section{Referencias}

Abad, María Cristina \& Bravo-Urquiza, Francisco (2012). El papel del consejo de administración en la gestión de la información de carácter previsional. Revista AECA, Asociación Española de Contabilidad y Administración de Empresas, 97, 18-21. Disponible en: http://www.aeca1.org/ revistaeca/revista97/97.pdf

Abed, Suzan; Al-Okdeh, Saleh \& Nimer, Khalil (2011). The Inclusion of Forecasts in the Narrative Sections of Annual Reports and their Association with Firms Characteristics: the Case of Jordan. International Business Research, 4 (4), 264-271. Disponible en: http://www.ccsenet.org/journal/index.php/ ibr/article/view/12387/8682

Accounting Standards Board, ASB (2006). Operating and Financial Review. Reporting Statement, RS. Kingston upon Thames, Surrey: ASB Publications. Disponible en: 
https://frc.org.uk/Our-Work/Publications/ ASB/Reporting-Statement-Operating-andFinancial-Review/Reporting-StatementThe-Operating-and-Financial-Re.aspx Accounting Standards Board, ASB (2007). A

Review of Narrative Reporting by UK Listed Companies in 2006. London: The Financial Reporting Council Limited. Disponible en: https://frc.org.uk/Our-Work/Publications/ ASB/Review-of-Narrative-Reporting-2007/ A-Review-of-Narrative-Reporting-by-UKListed-Compa.pdf

Accounting Standards Board, ASB (2009a).

Rising to the Challenge. A Review of Narrative Reporting by UK Listed Companies. London: The Financial Reporting Council Limited. Disponible en: https:/www.frc.org.uk/ Our-Work/Publications/ASB/Rising-to-theChallenge/Rising-to-the-challenge.aspx Accounting Standards Board, ASB (2009b).

\section{A Review of Narrative Reporting by UK} Listed Companies in 2008/2009. London: The Financial Reporting Council Limited. Disponible en: http://www.iasplus.com/en/ binary/uk/0910narrativereportingfull.pdf

Aerts, Walter (2001). Inertia in the

Attributional Content of Annual Accounting Narratives. The European Accounting Review, 10 (1), 3-32.

Ahmed, Kamran \& Courtis, John K. (1999).

Associations between Corporate Characteristics and Disclosure Levels in Annual Reports: A Meta-Analysis. The British Accounting Review, 31, 35-61. Aljifri, Khaled \& Hussainey, Khaled (2007). The Determinants of Forward-Looking Information in Annual Reports of UAE
Companies. Managerial Auditing Journal, 22 (9), 881-894.

American Institute of Certified Public Accountants, AICPA (1994). Improving Business Reporting - A Customer Focus: Meeting the Information Needs of Investors and Creditors. A comprehensive Report of the Special Committee on Financial Reporting (The Jenkins Report). Disponible en: http://www.aicpa.org/InterestAreas/ FRC/AccountingFinancialReporting/

DownloadableDocuments/Jenkins\%20 Committee\%20Report.pdf American Institute of Certified Public Accountants, AICPA (2001). Statement on Standards for Attestation Engagement (SSAE) 10. Attestation Standards: Revision and Recodification. AT Section 701: Management's Discussion and Analysis. Disponible en: http://www.aicpa.org/Research/Standards/ AuditAttest/Pages/SSAE.aspx, http://www. aicpa.org/Research/Standards/AuditAttest/ DownloadableDocuments/AT-00701.pdf Association of British Insurers, ABI (2006).

Developments in Narrative Reporting: An ABI Position Paper. Disponible en: https://www. ivis.co.uk/media/5884/Developments-in-

Narrative-Reporting.pdf Association of Chartered Certified Accountants, ACCA (2010). Hitting the Notes, but what's the Tune? An International Survey of CFO's Views on Narrative Reporting. London: ACCA, Deloitte. Disponible en: http://www.accaglobal.com/content/dam/ acca/global/PDF-technical/narrativereporting/hitting_the_notes.pdf 
Association of Chartered Certified

Accountants, ACCA; Tomorrow's Company, h2glenfern \& Black Sun Plc. (2006). Visions of the Future "to the Extent Necessary": the Debate on the Future of Narrative Reporting, June (2006).

Balata, Pascal \& Breton, Gaétan (2005). Narratives vs. Numbers in the Annual Report: Are They Giving the Same Message To The Investors? Review of Accounting and Finance, 4 (2), 5-25.

Banghøj, Jesper \& Plenborg, Thomas (2008). Value Relevance of Voluntary Disclosure in the Annual Report. Accounting and Finance, 48 (2), 159-180.

Barron, Orie E.; Kile, Charles O.; O'Keefe, Terrence B. (1999). MD\&A Quality as Measured by the SEC and Analysts' Earnings Forecasts. Contemporary Accounting Research, 16 (1), 75-109.

Beattie, Vivien (2005). Moving the Financial Accounting Research Front Forward: the UK Contribution. The British Accounting Review, 37 (1), 85-114

Beattie, Vivien; McInnes, Bill \& Fearnley, Stella (2004). Through the Eyes of Management: Narrative Reporting across Three SectorsFinal Report. London: Institute of Chartered Accountants in England and Wales, ICAEW, Centre for Business Performance.

Black Sun (2009). 100/08, New Challenge New Approach: Annual Analysis of FTSE100 Corporate Reporting 2008.

Botosan, Christine A. (1997). Disclosure Level and the Cost of Equity Capital. The Accounting Review, 72 (3), 323-349.
Botosan, Christine A. \& Plumlee, Marlene A. (2002). A Re-examination of Disclosure Level and the Expected Cost of Equity Capital. Journal of Accounting Research, 40 (1), 21-40.

Bozzolan, Saverio; Trombetta, Marco \& Beretta, Sergio (2009). Forward-Looking Disclosures, Financial Verifiability and Analysts' Forecasts: A Study of Cross-Listed European Firms. European Accounting Review, 18 (3), 435-473.

Bravo-Urquiza, Francisco; Abad-Navarro,

María Cristina \& Trombetta, Marco (2009). Disclosure Indices Design: Does it Make a Difference? Spanish Accounting Review, 12 (2), 253-277. Disponible en: http://dialnet.unirioja.es/servlet/ articulo?codigo $=3161648$ Bryan, Stephen H. (1997). Incremental Information Content of Required Disclosures Contained in Management Discussion and Analysis. The Accounting Review, 72 (2), 285-301.

Canadian Institute of Chartered Accountants, CICA (2002). Management's Discussion and Analysis: Guidance on Preparation and Disclosure. Toronto: Canadian Institute of Chartered Accountants, CICA.

Canadian Securities Administrators, CSA (2009). Guidance Regarding the Application of Forward-Looking Information Requirements under National Instrument 51-102 Continuous Disclosure Obligations. Staff Notice 51330. Disponible en: http://www.osc.gov. on.ca/documents/en/Securities-Category5/ csa_20091120_51-330_forward-looking.pdf 
Chartered Accountants of Canada, CA (2008a). CFO Beyond-GAAP Briefing. ForwardLooking Information. Toronto: Chartered Accountants of Canada, CA. Disponible en: http://www.cica.ca/publications/list-ofpublications/manual/item12824.pdf

Chartered Accountants of Canada, CA (2008b). 20 Questions Directors Should Ask about Management's Discussion and Analysis. $2^{\text {nd }}$ edition. Toronto: Chartered Accountants of Canada, CA. Disponible en: http://www. cica.ca/focus-on-practice-areas/governancestrategy-and-risk/directors-series/20question-series/item60595.pdf

Chartered Accountants of Canada, CA (2008c). MD\&A Disclosures in Volatile and Uncertain Times. CPR Alert, Canadian Performance Reporting, 8, 1-4. Disponible en: http://www.cica.ca/focus-on-practiceareas/reporting-and-capital-markets/ performance-reporting-resource-centre/ item13069.pdf

Chartered Accountants of Canada, CA (2009a).

Management's Discussion and Analysis: Guidance on Preparation and Disclosure. Comprehensive revision. Toronto: Canadian Institute of Chartered Accountants, CICA. Disponible en: http://www.cica.ca/ publications/list-of-publications/manual/ item29637.pdf

Chartered Accountants of Canada, CA (2009b). MD\&A Disclosures in Volatile and Uncertain Times. Volume 2: Analyzing Change and Liquidity. CPR Alert, Canadian Performance Reporting, 10, 1-4. Disponible en: http:// www.cica.ca/focus-on-practice-areas/ reporting-and-capital-markets/performancereporting-resource-centre/item13072.pdf

Chartered Institute of Management Accountants, CIMA; PricewaterhouseCoopers, PwC; Radley Yeldar \& Tomkins (2006). Report leadership: corporate reporting rethought. Effective. Disponible en: http://www.pwc.com/gx/en/auditservices/corporate-reporting/assets/pdfs/ reportleadershipfINAL.pdf

Chlala, Nadi \& Girard, Aline (1993). Preparing Management Reports. Certified Management Accountants, CMA Magazine, 67 (3), 31-35.

Clarkson, Peter M.; Kao, Jennifer L. \& Richardson, Gordon D. (1994). The Voluntary Inclusion of Forecasts in the MD\&A Section of Annual Reports. Contemporary Accounting Research, 11 (1), 423-450.

Clarkson, Peter M.; Kao, Jennifer L. \& Richardson, Gordon D. (1999). Evidence that Management Discussion and Analysis (MD\&A) is a Part of a Firm's Overall Disclosure Package. Contemporary Accounting Research, 16 (1), 111-134.

Clatworthy, Mark Anthony \& Jones, Michael J. (2003). Financial Reporting of Good News and Bad News: Evidence from Accounting Narratives. Accounting and Business Research, 33 (3), 171-185.

Clatworthy, Mark A. \& Jones, Michael J. (2006). Differential patterns of textual characteristics and company performance in the chairman's statement, Accounting, Auditing \& Accountability Journal, 19 (4), 493-511. 
Collins, Daniel W.; Kothari, S. P.; Shanken, Jay \& Sloan, Richard G. (1994). Lack of Timeliness and Noise as Explanations for the Low Contemporaneous ReturnEarnings Association. Journal of Accounting and Economics, 18, 289-324.

Comisión Nacional del Mercado de Valores, CNMV (2013). Guía para la elaboración del informe de gestión de las sociedades cotizadas. Disponible en: http://www.cnmv.es/ DocPortal/Publicaciones/Grupo/gestion_ ES_Web.pdf

Company Law Review Steering Group, CLRSG, Department for Business, Enterprise \& Regulatory Reform, UK Government (2000). Modern Company Law for a Competitive Economy: Developing the Framework. March, URN00/656. Disponible en: http://webarchive. nationalarchives.gov.uk/20121029131934/ http://www.berr.gov.uk/whatwedo/ businesslaw/co-act-2006/clr-review/ page25086.html

Copeland, Thomas (1978). Efficient Capital Markets: Evidence and Implications for Financial Reporting. Journal of Accounting, Auditing and Finance, 2 (1), 33-48.

Courtis, John K. (1998). Annual Report Readability of Annual Reports: Tests of the Obfuscation Hypothesis. Accounting, Auditing and Accountability Journal, 11 (4), 459-471.

Courtis, John K. (2004). Corporate Report Obfuscation: Artefact or Phenomenon. The British Accounting Review, 36 (3), 291-312.

Deloitte (2006). Write to Reason. Surveying OFRs and Narrative Reporting in Annual Reports.
London: Deloitte \& Touche LLP. Disponible en: http://www.deloitte.com/assets/ Dcom-UnitedKingdom/Local\%20Assets/ Documents/UK_Audit_Writetoreason_06. pdf

Deloitte (2007). Written to Order. Surveying OFR's, EBR's and Narrative Reporting in Annual Reports. London: Deloitte \& Touche LLP. Disponible en: http://www.deloitte. com/assets/Dcom-UnitedKingdom/ Local\%20Assets/Documents/UK_Audit_ WrittentoOrder_Nov2007(1).pdf Deloitte (2008). Write from the Start. Surveying Narrative Reporting in Annual Reports. London: Deloitte \& Touche LLP. Disponible en: http://www.deloitte.com/assets/ Dcom-UnitedKingdom/Local\%20Assets/ Documents/UK_Audit_Write_from_the_ start.pdf

Deloitte (2009). A Telling Performance. Surveying Narrative Reporting in Annual Reports. London: Deloitte LLP. Disponible en https://www.deloitte.com/assets/ Dcom-UnitedKingdom/Local\%20Assets/ Documents/Services/Audit/Corporate $\% 20$ Governance/UK_Audit_A_telling_ performance.pdf

Deloitte (2010). Swimming in Words. Surveying Narrative Reporting in Annual Reports. London: Deloitte LLP. Disponible en: https://www.deloitte.com/assets/DcomUnitedKingdom/Local\%20Assets/ Documents/Services/Audit/Corporate $\% 20$ Governance/UK_Audit_Swimming_in_ words.pdf

Deloitte (2011). Gems and Jetsam. Surveying Narrative Annual Reports. London: Deloitte 
LLP. Disponible en: http://www.deloitte. com/assets/Dcom-UnitedKingdom/ Local\%20Assets/Documents/Services/ Audit/uk-audit-gem-and-jetsam-lowresolution.pdf

Department for Business, Innovation and Skills, DBIS (2010, December). The Future of Narrative Reporting. A Consultation. London: Department for Business, Innovation and Skills, Crown Copyright, UK Government. Disponible en: https:// www.gov.uk/government/uploads/system/ uploads/attachment_data/file/31440/101057-future-narrative-reportingconsultation.pdf

Department for Business, Innovation and Skills, DBIS (2011, September). The Future of Narrative Reporting. Consulting on a New Reporting Framework. London: Department for Business, Innovation and Skills, Crown Copyright, UK Government. Disponible en: https://www.gov.uk/government/ uploads/system/uploads/attachment_data/ file/31402/11-945-future-of-narrativereporting-consulting-new-framework.pdf Department of Trade and Industry, DTI, United Kingdom (2004, May). The Operating and Financial Review. Practical Guidance for Directors.

Deutsches Rechnungslegungs Standards Committee, DRSC (2006). Comments on Management Commentary. Discussion paper. Disponible en: http://www.ifrs.org/CurrentProjects/IASB-Projects/ManagementCommentary/DP05/Comment-Letters/ Documents/16_230_MCCL19.pdf
Eikner, A. Eliane; Hefzi, Hassan \& Glezen, G. William (2000). Prospective Information in Managements' Discussion and Analysis: A Test of Incremental Information Content. The Journal of Interdisciplinary Studies, 13, 13-22. Disponible en: http://www. csupomona.edu/ jis/2000/eikner.pdf

Enhanced Business Reporting Consortium, EBRC (2005). The Enhanced Business Reporting Framework. EBR360, www.ebr360.org, http:// www.aicpa.org/InterestAreas/FRC/ AccountingFinancialReporting/ EnhancedBusinessReporting/pages/ enhancedbusinessreportingconsortium. aspx

Financial Accounting Standards Board, FASB (1978). Concept Statement 1: Objectives of Financial Reporting by Business Enterprises. Financial Accounting Standards Board, FASB (2001). Improving Business Reporting: Insights into Enhancing Voluntary Disclosures. Disponible en: http://www.fasb.org/cs/Cont entServer? $\mathrm{c}=$ Document_C\&pagename $=\mathrm{FA}$ SB\%2FDocument_C\%2FDocumentPage\&c $\mathrm{id}=1176156460184$

Financial Accounting Standards Board, FASB (2006). Preliminary Views: Conceptual Framework for Financial Reporting: Objective of Financial Reporting and Qualitative Characteristics of Decision-Useful Financial Reporting Information. Financial Accounting Series (1260-001, July 6). Norwalk, Connecticut: Financial Accounting Standards Board, FASB. Disponible en: http://www.fasb.org/jsp/FASB/Document_C/ 
DocumentPage $?$ cid $=1218220340119 \&$ accep tedDisclaimer $=$ true

Financial Accounting Standards Board, FASB (2008). An Improved Conceptual Framework for Financial Reporting. Chapter 1: The Objective of Financial Reporting. Exposure Draft. Disponible en: http://www.ifrs. org/Current-Projects/IASB-Projects/ Conceptual-Framework/EDMay08/ Documents/conceptual_framework exposure_draft.pdf

Financial Accounting Standards Board, FASB (2010). Conceptual Framework. Statement of Financial Accounting Concepts 8. Disponible en: http://www.fasb.org/jsp/FASB/ Document_C/DocumentPage?cid $=1176157$ 498129\&acceptedDisclaimer $=$ true

Financial Reporting Council, FRC (2008). The Combined Code on Corporate Governance. London: Financial Reporting Council, FRC. Disponible en: https://www.frc.org. uk/getattachment/1a875db9-b06e-44538f65-358809084331/The-Combined-Codeon-Corporate-Goverance.aspx

Financial Reporting Council, FRC (2009). Louder than Words. Principles and Actions for Making Corporate Reports less Complex and More Relevant. London: Financial Reporting Council, FRC. Disponible en: https://frc. org.uk/getattachment/7d952925-74ea-4debb659-e9242b09f2fa/Louder-than-words. aspx

Financial Reporting Council, FRC (2013). Exposure Draft: Guidance on the Strategic Report. Kingston upon Thames, Surrey: Financial Reporting Council, FRC. Disponible en: https://www.frc.org.uk/
Our-Work/Publications/Accounting-andReporting-Policy/Exposure-Draft-Guidanceon-the-Strategic-Report-File.pdf

Financial Reporting Review Panel, FRRP (2010). Annual Report 2010. London: Financial Reporting Council, FRC. Disponible en: https://www.frc.org.uk/ Our-Work/Publications/FRRP/AnnualReport-2010.pdf

Flöstrand, Per \& Ström, Niklas (2006). The Valuation Relevance of Non-Financial Information. Management Research News, 29 (9), 580-597.

Gelb, David; Holtzman, Mark P. \& Mest, David (2008). International Operations and Voluntary Disclosures by U.S. Based Multinational Corporations. Advances in International Accounting, 24 (2), 243-249.

Gelb, David S. \& Zarowin, Paul (2002). Corporate Disclosure Policy and the Informativeness of Stock Prices. Review of Accounting Studies, 7 (1), 33-52. Disponible en: http://pages.stern.nyu.edu/ pzarowin/ publications/P16_Corporate_disclosure_1. pdf

German Accounting Standards Board, GASB (2005). Management Reporting, German Accounting Standard (GAS) No. 15.

Group of 100, G100 (2003). Guide to Review of Operations and Financial Condition. Melbourne: Group of 100. Disponible en: http://www.group100.com.au/publications/ g100_Review_Operations2003.pdf

Healy, Paul M.; Hutton, Amy P. \& Palepu, Krishna G. (1999). Stock Performance and Intermediation Surrounding Sustained 
Increases in Disclosure. Contemporary Accounting Research, 16 (3), 485-520.

Hossain, Mahmud; Ahmed, Kamran \& Godfrey, Jayne M. (2005). Investment Opportunity set and Voluntary Disclosure of Prospective Information: A Simultaneous Equations Approach. Journal of Business Finance \& Accounting, 32 (5-6), 871-907.

Hussainey, Khaled \& Aal-Eisa, Jinan (2009). Disclosure and Dividend Signalling when Sustained Earnings Growth Declines. Managerial Auditing Journal, 24 (5), 445454.

Hussainey, Khaled; Schleicher, Thomas \& Walker, Martin (2003). Undertaking LargeScale Disclosure Studies when AIMR-FAF Ratings are Not Available: the Case of Prices Leading Earnings. Accounting and Business Research, 33 (4), 275-294.

Hussainey, Khaled \& Walker, Martin (2009). The Effects of Voluntary Disclosure and Dividend Propensity on Prices Leading Earnings. Accounting and Business Research, 39 (1), 275-294.

Independent Audit Limited (2006). Telling the story... through the eyes of the board. A framework for the Business Review.

Institute of Chartered Accountants in England and Wales, ICAEW (2003a). Prospective Financial Information. Guidance for UK Directors. London: Institute of Chartered Accountants in England and Wales, ICAEW. Disponible en: http://www.icaew.com/ / media/Files/Technical/Financial-reporting/ Information\%20for\%20better\%20markets/ IFBM/PFI.pdf
Institute of Chartered Accountants in England and Wales, ICAEW (2003b). The Operating and Financial Review and Human Capital Reporting. Is the OFR the Place for Human Capital Reporting? London: Institute of Chartered Accountants in England and Wales, ICAEW. Disponible en: http:// www.icaew.com/ /media/Files/Technical/ Research-and-academics/publications-andprojects/financial-reporting-publications/ the-operating-and-financial-review-andhuman-capital-reporting.pdf Instituto de Contabilidad y Auditoría de Cuentas, ICAC (2002). Libro Blanco para la Reforma de la Contabilidad en España. Madrid: Instituto de Contabilidad y Auditoría de Cuentas, ICAC. Disponible en: http://inza.files.wordpress.com/2013/09/ icac-libro-blanco-contabilidad.pdf International Accounting Standards Board, IASB (2001). Marco conceptual.

International Accounting Standards Board, IASB (2005). Management Commentary. Discussion paper. London: International Accounting Standards Board, IASB. Disponible en: http://www.ifrs.org/CurrentProjects/IASB-Projects/ManagementCommentary/DP05/Documents/ DPManagementCommentary.pdf International Accounting Standards Board, IASB (2009). Management Commentary. Exposure Draft. London: International Accounting Standards Board, IASB. Disponible en: http://www.ifrs.org/CurrentProjects/IASB-Projects/ManagementCommentary/ED09/Documents/ EDManagementCommentary.pdf 
International Accounting Standards Board, IASB (2010). Management Commentary. IFRS Practice Statement. London: International Accounting Standards Board, IASB. Disponible en: http:// www.ifrs.org/Current-Projects/IASBProjects/Management-Commentary/ IFRS-Practice-Statement/Documents/ Mana gementcommentarypractices tatement8December.pdf

International Accounting Standards Board, IASB (2013). A Review of the Conceptual Framework. Discussion Paper. London: International Accounting Standards Board, IASB. Disponible en: http://www. ifrs.org/Current-Projects/IASB-Projects/ Conceptual-Framework/Discussion-PaperJuly-2013/Documents/Discussion-PaperConceptual-Framework-July-2013.pdf International Federation of Accountants, IFAC (2007). Basis for Conclusions: International Standard on Auditing (ISA) 720. The Auditor's Responsibilities Relating to Other Information in Documents Containing Audited Financial Statements. Disponible en: http://www.ifac. org/sites/default/files/downloads/Basis_for_ Conclusions_ISA_720_Redrafted.pdf International Federation of Accountants, IFAC (2012). International Standard on Assurance Engagement (ISAE) 3400. The Examination of Prospective Financial Information. Disponible en: http:/www.ifac.org/sites/default/files/ publications/files/B006\%202013\%20 IAASB\%20Handbook\%20ISAE\%203400.pdf International Organization of Securities Commissions, IOSCO (2003). General Principles Regarding Disclosure of
Management Discussion and Analysis of Financial Condition and Results of Operations. Disponible en: http://www.iosco.org/ library/pubdocs/pdf/IOSCOPD141.pdf International Integrated Reporting Committee, IIRC (2013). The International Framework. Disponible en: http:/www.theiirc.org/ international-ir-framework/ Jones, Michael J. \& Shoemaker, Paul A. (1994). Accounting Narratives: A Review of Empirical Studies of Content and Readability. Journal of Accounting Literature, 13, 142-184.

Kent, Pamela \& Ung, Karen (2003). Voluntary Disclosure of Forward-Looking Information in Australia. Australian Journal of Management, 28 (3), 273-285.

Lang, Mark H. \& Lundholm, Russell J. (1996). Corporate Disclosure Policy and Analyst Behavior. The Accounting Review, 71 (4), 467-492.

Li, Feng (2008). Annual Report Readability, Current Earnings and Earnings Persistence. Journal of Accounting and Economics, 45, 221-247. Disponible en: https://www3. nd.edu/ carecob/April2010Conference/ Li\%20JAE\%202008.pdf

Li, Feng (2010). The Information Content of Forward-Looking Statements in Corporate Filings. A Naïve Bayesian Machine Learning Approach. Journal of Accounting Research, 48 (5), 1049-1102.

Lundholm, Russell J. \& Myers, Linda A. (2002). Bringing the Future Forward: the Effect of Disclosure on the Returns-Earnings Relation. Journal of Accounting Research, 40 (3), 809-839. 
Mallett, Richard \& Slynn, Richard (2005). The Operating and Financial Review. Financial Management, September, 35-36.

Mallo-Rodríguez, Carlos (1990). Informe de gestión e imagen empresarial. Revista de Economía, Consejo General de Colegios de Economistas de España, 6, 38-41.

Marston, Claire L. \& Shrives, Philip J. (1991). The Use of Disclosure Indices in Accounting Research. The British Accounting Review, 23 (3) 195-210.

Muslu, Volkan; Radhakrishnan, Suresh; Subramanyam, K. R. \& Lim, Dongkuk (2011). Firm's Information Environment and Forward-Looking Disclosures in the MD\&A. University of Texas at Dallas and University of Southern California, Working paper.

Muslu, Volkan; Radhakrishnan, Suresh; Subramanyam, K. R. \& Lim, Dongkuk (2014). Forward-Looking MD\&A Disclosures and the Information Environment. Management Science, Forthcoming, working paper.

Ontario Securities Commission, OSC (2008). Corporate Finance Branch Report 2008. Staff Notice 51-706. Disponible en: http://www. osc.gov.on.ca/documents/en/SecuritiesCategory5/sn_20101020_51-706_cfrpt-2010.pdf

Ontario Securities Commission, OSC (2013). Forward-Looking Information Disclosure. Staff Notice 51-721. Disponible en: https://www. osc.gov.on.ca/documents/en/SecuritiesCategory5/sn_20130613_51-721_forwardlooking.pdf

Orens, Raf \& Lybaert, Nadine (2007). Does the Financial Analysts' Usage of Non-Financial
Information Influence the Analysts' Forecast Accuracy? Some Evidence from the Belgian Sell-Side Financial Analyst. The International Journal of Accounting, 42 (3), 237-271.

Organización para la Cooperación y el Desarrollo Económicos, OCDE (2004). Principios de gobierno corporativo de la OCDE. París: Organización para la Cooperación y el Desarrollo Económicos, OCDE. Disponible en: http://www.oecd.org/daf/ca/ corporategovernanceprinciples/37191543. pdf

O'Sullivan, Madonna; Percy, Majella \& Stewart, Jenny (2008). Australian Evidence on Corporate Governance Attributes and their Association with Forward-Looking Information in the Annual Report. Journal of Management \& Governance, 12 (1), 5-35. PricewaterhouseCoopers, $\mathrm{PwC}$ (2007). Guide to Forward-Looking Information. Don't Fear the Future: Communicating with Confidence. London: PricewaterhouseCoopers, PwC. Disponible en: http://www.pwc.com/gx/en/ audit-services/corporate-reporting/assets/ pdfs/860-global-forward-looking-guide.pdf PricewaterhouseCoopers, PwC (2008). Joining the Dots. A Summary of the Narrative Reporting Practices of the FTSE 350. London: PricewaterhouseCoopers, $\mathrm{PwC}$. Disponible en: http://pwc.blogs.com/ corporatereporting/files/ftse_350_survey.pdf PricewaterhouseCoopers, PwC (2009). A Snapshot of FTSE 350 Reporting. London: PricewaterhouseCoopers, PwC. Disponible en: http://pwc.blogs.com/files/a-snapshotof-ftse-350-reporting.pdf 
Radley Yeldar (2008a). Narrative Reporting Content in the FTSE 100. How Does it Stack Up? On and Offline 2008.

Radley Yeldar (2008b). Challenges Ahead.

Shaping the Future of Investor Communication.

Schleicher, Thomas (2012). When is Good

News Really Good News? Accounting and

Business Research, 42 (5), 547-573.

Schleicher, Thomas; Hussainey, Khaled

\& Walker, Martin (2007). Loss Firms'

Annual Report Narratives and Share

Price Anticipation of Earnings. The British

Accounting Review, 39 (2), 153-171.

Schleicher, Thomas \& Walker, Martin (1999).

Share Price Anticipation of Earnings and

Management's Discussion of Operations

and Financing. Accounting and Business

Research, 29 (4), 321-335.

Schleicher, Thomas \& Walker, Martin (2010).

Bias in the Tone of Forward-Looking

Narratives. Accounting and Business

Research, 40 (4), 371-490.

Schroeder, Richard G.; Clark, Myrtle W. \&

Cathey, Jack M. (2005). Financial Accounting Theory and Analysis. Text Readings and Cases.

Hoboken, New Jersey: Wiley.

Sengupta, Partha (1998). Corporate Disclosure

Quality and the Cost of Debt. The

Accounting Review, 73 (4), 459-474.

Sydserff, Robert \& Weetman, Pauline (1999).

A Texture Index for Evaluating Accounting

Narratives. An Alternative to Readability

Formulas. Accounting, Auditing \&

Accountability Journal, 12 (4), 459-488.

Sydserff, Robert \& Weetman, Pauline (2002).

Developments in Content Analysis: A

Transitivity Index and DICTION Scores.
Accounting, Auditing \& Accountability Journal, 15 (4), 523-544.

Tarca, Ann; Street, Donna L. \& Aerts, Walter (2011). Factors Affecting MD\&A Disclosures by SEC Registrants: views of practitioners. Journal of International Accounting Auditing and Taxation, 20 (1), 45-59.

Tomorrow's Company (2007). The Future of Corporate Reporting. State of Play-February 2007. Welker, Michael (1995). Disclosure Policy, Information Asymmetry and Liquidity in Equity Markets. Contemporary Accounting Research, 11 (2), 801-827.

Yeoh, Peter (2010). Narrative Reporting: the UK Experience. International Journal of Law and Management, 52 (3), 211-231.

\section{Legislación}

Canadá. Canadian Securities Administrators, CSA (2007). Management's Discussion and Analysis. National Instrument (NI) Form 51-102 Continuous Disclosure Obligations. Disponible en: https://www.osc.gov.on.ca/ documents/en/Securities-Category5/ rule_20111031_51-102_unofficialconsolidation-post-ifrs.pdf

España. Código de Comercio, 16 de octubre de 1885. Boletín Oficial del Estado, BOE, 289, 16 de octubre de 1885. Disponible en: https:// www.boe.es/datos/pdfs/BOE/1885/289/ R00169-00613.pdf. Modificado por la Ley 19/1989. Boletín Oficial del Estado, BOE, 178, 27 de julio de 1989. Disponible en: https:// www.boe.es/boe/dias/1989/07/27/pdfs/ A24085-24110.pdf. Ley 24/2001. Boletín Oficial del Estado, BOE, 313, 31 de diciembre de 2001. Disponible en: http://www.boe.es/ 
boe/dias/2001/12/31/pdfs/A50493-50619. pdf. Ley 34/2002. Boletín Oficial del Estado, BOE, 166, 12 de julio de 2002. Disponible en: http://www.boe.es/boe/dias/2002/07/12/ pdfs/A25388-25403.pdf. Ley 22/2003. Boletín Oficial del Estado, BOE, 164, 10 de julio de 2003. Disponible en: http://www. boe.es/boe/dias/2003/07/10/pdfs/A2690526965.pdf. Ley 62/2003. Boletín Oficial del Estado, BOE, 313, 31 de diciembre de 2003. Disponible en: http://www.boe.es/boe/ dias/2003/12/31/pdfs/A46874-46992.pdf. Ley 2/2007. Boletín Oficial del Estado, BOE, 65, 16 de marzo de 2007. Disponible en: http://www.boe.es/boe/dias/2007/03/16/ pdfs/A11246-11251.pdf. Ley 16/2007. Boletín Oficial del Estado, BOE, 160, 5 de julio de 2007. Disponible en: http://www.boe.es/boe/ dias/2007/07/05/pdfs/A29016-29047.pdf

España. Real Decreto Legislativo 1/2010, texto refundido de la Ley de sociedades de capital. Boletín Oficial del Estado, BOE, 161, 3 de julio de 2010. Disponible en: http://www.boe.es/ diario_boe/txt.php?id=BOE-A-2010-10544

Estados Unidos. Securities and Exchange Commission, SEC (1980). Amendments to Annual Report Form, Related Forms, Rules, Regulations and Guides: Integration of Securities' Acts Disclosure System. Securities Act Release 33-6234, 45 FR 6368.

Estados Unidos. Securities and Exchange Commission, SEC (1982). Regulation S-K, Item 303: Management's Discussion and Analysis of Financial Condition and Results of Operations. Disponible en: http://www.gpo. gov/fdsys/pkg/CFR-2001-title17-vol2/xml/ CFR-2001-title17-vol2-sec229-303.xml
Estados Unidos. Securities and Exchange Commission, SEC (1989). Interpretation: Management's Discussion and Analysis of Financial Condition and Results of Operations; Certain Investment Company Disclosures. Securities Act Release release 6835, 54 FR 22427. Disponible en: http://www.sec.gov/ rules/interp/33-6835.htm

Estados Unidos. Securities and Exchange Commission, SEC (1992). Exchange Act Release 34-30532, Accounting and Auditing Enforcement Release AE-363 (March 31, 1992).

Estados Unidos. Securities and Exchange Commission, SEC (1998). Exchange Act Release 34-40305, Accounting and Auditing Enforcement Release AE-1061. (August 5, 1998). Disponible en: https://www.sec.gov/ litigation/admin/3440305.txt

Estados Unidos. Securities and Exchange Commission, SEC (2003). Interpretation: Commission Guidance Regarding Management's Discussion and Analysis of Financial Condition and Results of Operations. September (Release 33-8350; 34-48960; FR-72). Disponible en: http://www.sec.gov/ rules/interp/33-8350.htm

Estados Unidos. Securities and Exchange Commission, SEC (2005). Accounting and Auditing Enforcement Release 2232 (April 18, 2005). Disponible en: http://www.sec.gov/ litigation/admin/33-8569.pdf

Reino Unido. Companies Act 1985, Part VII:

Accounts and Audit, Chapter I, Director's Report, Section 234. Disponible en: http://www. legislation.gov.uk/ukpga/1985/6/contents 
Reino Unido. Companies Act 1985, Regulations 2005 (Statutory Instrument $2005 N^{\circ}$ 1011, SI 2005/1011).

Reino Unido. Companies Act 2006, Part 15 Accounts and reports, Directors' report, sections 415-419. Disponible en: http://www. legislation.gov.uk/ukpga/2006/46/pdfs/ ukpga_20060046_en.pdf

Reino Unido. Companies Act 2006, Regulations 2013 (Strategic Report and Director's Report).

Unión Europea. Directiva 2003/51/CE del Parlamento Europeo y del Consejo, de 18 de junio de 2003, por la que se modifican las Directivas 78/660/CEE, 83/349/CEE, 86/635/CEE y 91/674/CEE del Consejo sobre las cuentas anuales y consolidadas de determinadas formas de sociedades, bancos $y$ otras entidades financieras y empresas de seguros. Disponible en: https://www.boe.es/ doue/2003/178/L00016-00022.pdf

Unión Europea. Directiva 2004/25/CE del Parlamento Europeo y del Consejo, de 21 de abril de 2004, relativa a las ofertas públicas de adquisición. Disponible en: https:/www. boe.es/doue/2004/142/L00012-00023.pdf Unión Europea. Directiva 2004/109/CE del Parlamento Europeo y del Consejo, de 15 de diciembre de 2004, sobre la armonización de los requisitos de transparencia relativos a la información sobre los emisores cuyos valores se admiten a negociación en un mercado regulado y por la que se modifica la Directiva 2001/34/CE. Disponible en: http://www.boe. es/doue/2004/390/L00038-00057.pdf

Unión Europea. Directiva 2006/46/CE del Parlamento Europeo y del Consejo, de 14 de junio de 2006, por la que se modifican las Directivas del Consejo 78/660/CEE relativa a las cuentas anuales de determinadas formas de sociedad, 83/349/CEE relativa a las cuentas consolidadas, 86/635/CEE relativa a las cuentas anuales y a las cuentas consolidadas de los bancos y otras entidades financieras $y$ 91/674/CEE relativa a las cuentas anuales $y$ a las cuentas consolidadas de las empresas de seguros. Disponible en: http://eur-lex. europa.eu/legal-content/EN/TXT/?qid=141 2199406987\&uri=CELEX:32006L0046

Unión Europea. Directiva 2013/34/UE del Parlamento Europeo y del Consejo, de 26 de junio de 2013, sobre los estados financieros anuales, los estados financieros consolidados $y$ otros informes afines de ciertos tipos de empresas, por la que se modifica la Directiva 2006/43/CE del Parlamento Europeo y del Consejo y se derogan las Directivas 78/660/ CEE y 83/349/CEE del Consejo. Disponible en: http://www.boe.es/doue/2013/182/ L00019-00076.pdf

- Fecha de recepción: 27 de febrero de 2014

- Fecha de aceptación: 8 de marzo de 2014

- Disponible en línea: 01 de julio de 2014

\section{Para citar este artículo}

Ibáñez-Jiménez, Eva María \& Muñoz-Merchante, Ángel (2014). Información financiera prospectiva: análisis comparado de su normalización y evidencia empírica. Cuadernos de Contabilidad, 15 (38), 459-500.

doi: 10.11144/Javeriana.cc15-38.ifpa 